\title{
Impact of Mobility on the BER Performance of Ad Hoc Wireless Networks
}

\author{
Gianluigi Ferrari, Member, IEEE, and Ozan K. Tonguz, Member, IEEE
}

\begin{abstract}
In this paper, the authors quantify the impact of mobility on the bit error rate (BER) performance of ad hoc wireless networks. Analytical expressions, relating the BER at the end of a multihop route with the mobility characteristics of the nodes and the switching strategy, are derived on the basis of a rigorous detection-theoretic approach. In particular, two network switching scenarios are considered: 1) opportunistic nonreservation-based switching (ONRBS), where a message flows from source to destination by opportunistically choosing the available shortest consecutive links and 2) reservation-based switching (RBS), where, after the creation of a multihop route from source to destination, the message is "forced" to flow over the reserved links, regardless of their actual lengths. The network performance is evaluated for both an ideal case (without interference) and a realistic case (with interference). The improved robustness against mobility offered by ONRBS, with respect to RBS, is analyzed and quantified. In particular, two node mobility models, known as directionpersistent (DP) and direction-non-persistent (DNP), are considered, and it is shown that DP mobility causes a much more profound degradation in the end-to-end route BER than DNP mobility. This conclusion is more pronounced in ad hoc wireless networks employing RBS. Overall, the results show that if the medium access control (MAC) protocol is not efficient in canceling or mitigating the interference, then the role of the switching/ routing strategy in network performance is quite minor.
\end{abstract}

Index Terms-Ad hoc wireless networks, internode interference (INI), mobility, nonreservation-based switching (NRBS), RBS.

\section{INTRODUCTION}

$\mathbf{M}$ ULTIHOP wireless ad hoc and sensor networks have recently attracted a lot of attention due to their potential to provide ubiquitous connectivity [1], [2]. In particular, in future generations of ad hoc wireless networks, nodes are likely to be mobile (e.g., car-based ad hoc wireless networks) [1], [3]-[5]. However, maintaining multihop communication routes is a challenge, especially in the case of mobile nodes: the topology is time-varying and, once a route has been established, local route maintenance is necessary in order for that route to continue to work when a link is broken [6]-[9]. In [10], it is shown that designed mobility might be helpful in surveillance sensor networks.

Manuscript received March 29, 2005; revised January 3, 2006 and February 17, 2006. This work was supported in part by an ARO Grant to Cylab of Carnegie Mellon University. The review of this paper was coordinated by Dr. W. Zhuang.

G. Ferrari is with the Department of Information Engineering, University of Parma, 43100 Parma, Italy (e-mail: gianluigi.ferrari@unipr.it).

O. K. Tonguz is with the Electrical and Computer Engineering Department, Carnegie Mellon University, Pittsburgh, PA 15213-3890 USA (e-mail: tonguz@ece.cmu.edu).

Color versions of one or more of the figures in this paper are available online at http://ieeexplore.ieee.org.

Digital Object Identifier 10.1109/TVT.2006.883790
Recently, the concept of transport capacity has been introduced and quantified, via an approach that is informationtheoretically inspired, in [11]. The concept of transport capacity, which simultaneously takes into account the amount of information transferred in the network and the distance over which the information is transferred, represents a very useful measure of the maximum sustainable information flow in multihop ad hoc wireless networks. While mobility can, theoretically speaking, increase the transport capacity (as shown in [12]), the effect of mobility on the performance of practical ad hoc wireless networks is deleterious [13]. In [14], Neely and Modiano consider a network with cell-partitioned structure, where nodes move according to a simplified uniform mobility model, and redundant packets are sent by each source to the corresponding destination through multiple paths. For this scenario, fundamental tradeoffs between network capacity and queuing delay are derived. In [15], the maximum transmission rate and an upper bound on the transmission delay are evaluated in an ad hoc wireless networking scenario with mobile nodes, taking into account the presence of fading.

A novel communication-theoretic framework for ad hoc wireless networks has been proposed in [16]-[19], on the basis of a "bottom-up" approach. In particular, the impact of the physical-layer characteristics on the network performance, jointly with the medium-access-control (MAC) protocol employed and the specific switching strategy (either reservationbased switching (RBS) [16], [17] or non-RBS (NRBS) with disjoint multihop routes [18]), is evaluated.

While in [16]-[18] a network-communication scenario with static nodes placed at the vertices of a regular square grid is considered, in this paper we extend the proposed framework by incorporating the effects of node mobility on the performance of ad hoc wireless networks. Rather than relying heavily on computer simulations, we propose a novel semianalytical approach for quantifying the impact of mobility. We consider both an ideal network-communication scenario, without internode interference (INI), and a realistic networkcommunication scenario, where communication is affected by INI. In the latter case, reserve-and-go (RESGO) MAC protocol, originally proposed in [16] and characterized by multihop route reservation and absence of collision-based retransmission in intermediate links, ${ }^{1}$ is used. Two possible switching strategies

\footnotetext{
${ }^{1}$ This MAC protocol was incorrectly referred to in [16] and [17] as the Aloha MAC protocol, for its resemblance, in terms of route activation being independent from the activity of other nodes in the network, with the classic Aloha MAC protocol [20]. However, there are significant differences that make the proposed protocol different from the classic Aloha MAC protocol: 1) multihop route reservation and 2) no use of retransmission techniques.
} 
are considered: 1) opportunistic NRBS (ONRBS), where successive hops from source to destination are dynamically chosen based on their lengths (for example, nodes could be equipped with some location-tracking devices able to evaluate the distance between them), and 2) RBS, where successive hops of a multihop route are activated consecutively regardless of their actual lengths. In both cases, we consider two different mobility models, known as direction-persistent (DP) and direction-nonpersistent (DNP), characterized by the fact that the directions of movement of the two nodes at the ends of a link do not change or change, respectively, during the transmission of a message. We point out, however, that the proposed framework can be used for any mobility pattern, provided that a statistical description of the mobility pattern is available. Numerical results, in terms of bit error rate (BER) at the end of a multihop route with an average number of hops, are presented to assess the performance of the considered ad hoc wireless networkcommunication schemes. In particular, in this paper, we evaluate the impact of 1) switching technique; 2) mobility pattern; 3) message length; and 4) maximum node speed. As expected, ONRBS mitigates the performance degradation, caused by node mobility, more efficiently than RBS. We also show that the impact of interference is deleterious, almost irrespective of the switching strategy. Therefore, if the MAC protocol is relatively simple (like RESGO), then the interference might be detrimental. Our results also show that mobility patterns characterized by frequent changes of direction lead to improved performance, and that this improvement is more pronounced in a scenario with RBS, rather than in a scenario with ONRBS. Finally, we draw some conclusions on the impact of the MAC protocol and switching (routing) strategy employed on the performance of ad hoc wireless networks.

The remainder of this paper is organized as follows. In Section II, we consider basic assumptions and preliminaries for the evaluation of the route BER in a scenario without interference. In Section III, a rigorous detection-theoretic approach to performance evaluation for scenarios with interference is proposed. In Section IV, we describe the principles of ONRBS and RBS in multihop ad hoc wireless networks. In Section V, the details of the two mobility models considered in this paper are outlined, and a semi-analytical approach to performance evaluation is proposed. In Section VI, extensive numerical results are presented that quantify the impact of node mobility, together with the switching strategy, on the network performance. Finally, conclusions are drawn in Section VII.

\section{Assumptions And Preliminaries}

The ad hoc wireless network-communication scenario considered in this paper can be characterized as follows.

1) Peer-to-peer multihop communications are considered.

2) The route creation phase is not considered. While this is a fundamental phase for ad hoc wireless networking [1], in this paper we are interested in analyzing the "steadystate" performance in the presence of node mobility. In the remainder of this paper, we do not comment on the route creation phase. Rather, we evaluate the network performance assuming that routes have been created. Obviously, mobility affects the route creation phase as well. However, assuming that an efficient routing protocol is employed, the duration of the route discovery phase should be significantly lower than the route utilization phase. Therefore, the impact of mobility is more significant in the transmission phase.

3) A node transmits information only after reserving a multihop route to its desired destination, and we assume that the generation/transmission process is described by a Poisson distribution, with parameter $\lambda$ (dimension: $[\mathrm{msg} / \mathrm{s}]$ ), corresponding to the average message generation rate. In particular, the messages have fixed length $M$ (dimension: $[\mathrm{b} / \mathrm{msg}]$ ), and the transmission data rate at each node, which is denoted as $R_{\mathrm{b}}$ (dimension: [b/s]), is fixed as well. Neglecting the propagation time, the duration of a message transmission between the two nodes of a link is $D_{\mathrm{msg}} \triangleq M / R_{\mathrm{b}}$. Each node, after transmitting a generated message, releases the reserved multihop route. Extensions to packetized transmissions can be considered based on the framework presented in [19].

4) The analysis of the delay incurred with multihop communications, although fundamental, is beyond the scope of this paper. In fact, we limit ourselves to the evaluation of a communication-theoretic performance metric such as the route BER. The analysis presented in this paper can be extended to take into account the delay performance, according to the approach proposed in [19], [21] and [22].

5) No intermediate retransmission mechanism is used. However, the proposed approach can be extended to this case by taking into account the impact of retransmissions on the link BER. We are currently investigating this research direction, and preliminary results are presented in [21], [22].

6) We assume that there are $N$ mobile nodes in the network, and that they are confined inside a network surface with area $A$. This may correspond, for example, to an ad hoc wireless network of laptops in a university campus (people are likely to move inside the campus, but they tend to remain within the campus area) or to an ad hoc wireless network of mobile phones and laptops in an airport terminal.

In order to derive an analytical model that captures the impact of node mobility, we first recall a few basic results from the framework proposed in [19]. In particular, it is assumed that $N$ nodes are placed at the vertices of a square grid over a circular surface with area $A$ : each node has, therefore, four nearest neighbors (neglecting border effects). Any multihop route is constituted by a sequence of hops between neighboring nodes. Denoting by $\rho_{S}=N / A$, the node spatial density, in [17] it is shown that the distance between two neighboring nodes, denoted by $d_{\text {link }}$, can be written as

$$
d_{\text {link }}=\frac{1}{\sqrt{\rho_{S}}} .
$$

Denoting by BER link $_{\text {the }}$ BER at the end of a single link, and assuming that 1 ) each link (of length $d_{\text {link }}$ ) is characterized 
by the same $\mathrm{BER}^{2}$; 2) there are no burst errors; and 3 ) the uncorrected bit errors in a link (after the processing at the receiving node of the link) are never recovered from in the following links, it is possible to show that the BER at the end of a multihop route with $n_{\mathrm{h}}$ hops, denoted by $\mathrm{BER}_{\text {route }}^{\left(n_{\mathrm{h}}\right)}$, can be expressed as [16]

$$
\mathrm{BER}_{\text {route }}^{\left(n_{\mathrm{h}}\right)}=1-\left(1-\mathrm{BER}_{\text {link }}\right)^{n_{\mathrm{h}}} .
$$

Expression (2) shows the dependence of the BER at the end of a multihop route on the number of hops $n_{\mathrm{h}}$ and on the link BER. In particular, the link BER depends, among other parameters, on the SNR at the receiving node of the link,

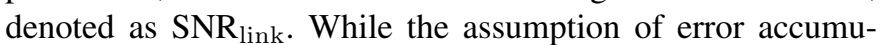
lation used to derive (2) might seem overly pessimistic, simulation results show that it is accurate for route BER values of interest.

In order to perform an average performance analysis, we evaluate the BER at the end of a multihop route with an average number of hops. In other words, we evaluate the BER expression (2) with an average number of hops $\bar{n}_{\mathrm{h}}$. It is possible to show that this represents an accurate estimate of the expected route BER, where the expectation is carried out over the number of hops [19]. A good statistical description of the number of hops $n_{\mathrm{h}}$ is given by a "quasi-binomial" distribution (obtained from a binomial distribution by eliminating the probability mass at zero) — this is motivated by the fact that very short or very long routes are more unlikely than routes with medium length. We therefore assume that the number of hops $n_{\mathrm{h}}$ is quasi-binomially distributed between one and the maximum number of hops, denoted as $n_{\mathrm{h}}^{\max }$, over a diameter of the circular network surface-it can be shown that $n_{\mathrm{h}}^{\max }=$ $2 r_{A} / d_{\text {link }}=\lfloor 2 \sqrt{N / \pi}\rceil$, where $r_{A}$ is the radius of the surface area (i.e., $A=\pi r_{A}^{2}$ ), and the notation $\left.\mid *\right\rceil$ indicates the integer value closest $\mathrm{t}^{3}$ to the argument $*$. Therefore, one obtains $\bar{n}_{\mathrm{h}}=$ $n_{\mathrm{h}}^{\max } / 2=\lfloor\sqrt{N / \pi}\rceil$. Finally, the BER at the end of a multihop route with an average number of hops can be written as

$$
\mathrm{BER}_{\text {route }}=\mathrm{BER}_{\text {route }}^{\left(\bar{n}_{\mathrm{h}}\right)}=1-\left(1-\mathrm{BER}_{\text {link }}\right)^{\bar{n}_{\mathrm{h}}} .
$$

We note that the assumption that the maximum number of hops corresponds to a diameter of the network circular surface is not precise, in the sense that a multihop route between two nodes at the end points of a diameter might be "around" the straight line connecting the nodes. However, assuming that the routing strategy is "intelligent," it is possible to show that $\bar{n}_{\mathrm{h}}=\Theta(\sqrt{N})$, regardless of the network surface shape, where the notation $y(n)=\Theta(x(n))$ indicates that $y(n)$ is "on the

\footnotetext{
${ }^{2}$ Assumption 1) might not be exactly true in a real communication scenario affected by INI, since the level of interference experienced by a node depends on its position with respect to the other nodes. However, the obtained results will provide useful insights into the impact of node mobility in more realistic scenarios as well.

${ }^{3}$ We point out that the notation $\lfloor x\rceil$ is ill-defined if the noninteger part of $x$ is 0.5 . However, since it is applied to real-valued quantities, the probability that the noninteger part is 0.5 is zero.
}

order" of $x(n)$ for $n \geq n_{0}$ [23]. In particular, all the results presented in the following have to be interpreted as results valid "on the order," rather than exact results. As such, they provide useful guidelines to understand the impact of mobility on ad hoc wireless networks.

We assume that the transmitted signal is simply affected by free-space loss. Hence, according to Friis formula [24], the received signal power at distance $d_{\text {link }}$ from the transmitter (i.e., the received signal power in a single link transmission), denoted as $P_{\mathrm{r}}$, can be expressed as

$$
P_{\mathrm{r}}=\frac{\alpha P_{\mathrm{t}}}{d_{\text {link }}^{2}}=\frac{G_{\mathrm{t}} G_{\mathrm{r}} c^{2} P_{\mathrm{t}}}{(4 \pi)^{2} f_{\mathrm{l}} f_{\mathrm{c}}^{2} d_{\mathrm{link}}^{2}}
$$

where $P_{\mathrm{t}}$ is the transmit power (assumed common for all nodes), $G_{\mathrm{t}}$ and $G_{\mathrm{r}}$ are the transmitter and receiver antenna gains, respectively, $f_{\mathrm{c}}$ is the carrier frequency, $c$ is the speed of light, and $f_{1} \geq 1$ is a loss factor. In the following, we consider $G_{\mathrm{t}}=G_{\mathrm{r}}=1$ (omnidirectional antennas) and $f_{\mathrm{l}}=1$ (no system losses not associated with propagation). While we consider here a scenario with free space propagation $\left(P_{\mathrm{r}} \propto P_{\mathrm{t}} / d_{\text {link }}^{2}\right)$, our approach can also be extended to other propagation scenarios characterized by stronger attenuation $\left(P_{\mathrm{r}} \propto P_{\mathrm{t}} / d_{\text {link }}^{\gamma}\right.$, where $\gamma>2$ ).

Note that in the case of an ad hoc wireless network with mobile nodes, owing to the assumption that the nodes, although mobile, are confined within the network surface (i.e., the average node spatial density remains fixed to $\rho_{S}=N / A$ ), it follows that, on average, any node can find a neighboring node at distance $\Theta\left(1 / \sqrt{\rho_{S}}\right)$ - provided that it can afford waiting a sufficiently long time.

In an ideal (no interference) scenario, the only noise affecting the received signal at each node is the thermal noise, which is modeled as additive, white, and Gaussian. We define the link SNR as

$$
\mathrm{SNR}_{\text {link }}=\frac{E_{\text {bit }}}{E_{\text {thermal }}}
$$

where $E_{\mathrm{bit}} \triangleq P_{\mathrm{r}} / R_{b}$ is the received energy per bit, and $E_{\text {thermal }}$ is the thermal noise power spectral density, which can be written as $F k T_{0}$, where $F$ is the noise figure [24], $k=1.38 \times 10^{-23} \mathrm{~J} / \mathrm{K}$ is the Boltzmann's constant, and $T_{0}$ is the room temperature $\left(T_{0}=300 \mathrm{~K}\right)$. Therefore, one can write

$$
\mathrm{SNR}_{\text {link }}=\frac{\alpha P_{\mathrm{t}}}{F k T_{0} R_{b} d_{\text {link }}^{2}} .
$$

The assumption of the absence of interference holds in a network-communication scenario where different multihop communication routes do not interfere with each other. This could be obtained, for example, by using perfectly orthogonal spreading codes (or disjoint frequency bands) in active disjoint multihop routes, or considering local scheduling in order to 
avoid collisions between nodes near each other during transmission. In the case of uncoded binary phase shift keying (BPSK), which will be the modulation format used in the remainder of this paper, the link BER is

$$
\begin{aligned}
\mathrm{BER}_{\text {link }} & =Q\left(\sqrt{2 \mathrm{SNR}_{\text {link }}}\right) \\
& =\frac{1}{\sqrt{2 \pi}} \int_{\sqrt{2 \mathrm{SNR}_{\text {link }}}}^{\infty} e^{-x^{2} / 2} \mathrm{dx} .
\end{aligned}
$$

The extension of this work to scenarios with other modulation formats can be carried out following the approach in [25]. Note also that the results in the ideal case may be representative for an ultrawideband network-communication scenario, where interference is practically negligible.

\section{INTERFERENCE ANALYSIS}

In order to characterize the BER performance in a scenario with interference, we distinguish between two different MAC protocols considered in this paper.

\section{A. RESGO MAC Protocol}

The main characteristic of RESGO MAC protocol is the fact that a node, after reserving a multihop route to its destination, starts immediately the transmission of a message, since there is no channel sensing. Hence, each node in the network can potentially and independently damage the transmission between the two nodes at the two ends of a link. Instead of considering the probability of collision between the transmitted message and an interfering message, we focus on the probability of a single bit in the transmitted message being interfered by the transmission from the other nodes in the network. This choice is motivated by the fact that in a wireless communication scenario, if two messages collide only partially, the overall quality of the received message might still be acceptable. For instance, in wired network communications, since the propagation loss is negligible, the absence of collision leads to virtually errorfree transmissions. Therefore, the network-communication protocols are often based on the use of retransmission mechanisms in order to avoid collisions in subsequent transmissions. Since the considered MAC protocols in this paper do not make use of retransmission mechanism retransmission mechanisms, a bit-level interference analysis is more accurate for a network-communication scenario with a quality of service (QoS) based on the maximum tolerable BER at the end of a communication route.

We now evaluate the interference experienced by the receiving node of an intermediate link of a multihop route. We will refer to the nodes forming this link as $n_{S}$ (transmitting) and $n_{D}$ (receiving), respectively. The vulnerable interval at a generic interfering node represents the interval such that any message transmission from this node, starting within this interval, will interfere with the considered bit in the message transmitted by the source node. It is possible to conclude that, in a scenario with RESGO MAC protocol, the length of the vulnerable interval does not depend on the specific bit position in the

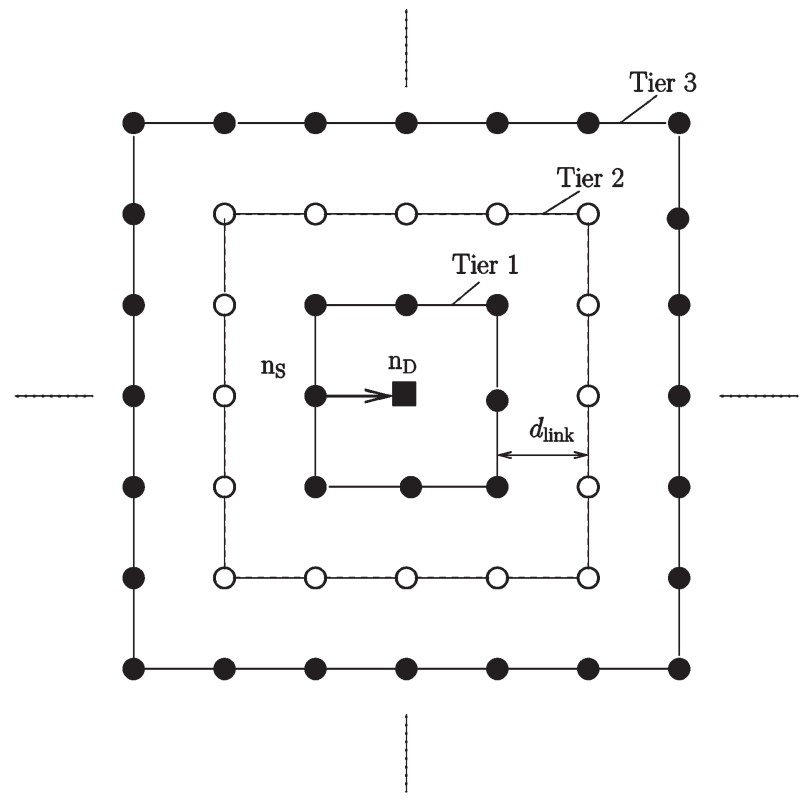

Fig. 1. Concentric tiered structure of nodes, in the case of square-grid topology, around node $n_{D}$.

transmitted message and is equal to $D_{\mathrm{msg}}$. Hence, the analysis on a per-bit basis does not depend either on the particular bit inside the transmitted message or on the particular interfering node. One thus obtains

$$
\begin{aligned}
p & \triangleq P\left\{\begin{array}{c}
\text { bit interference } \\
\text { from node } \mathrm{n}_{\ell}
\end{array}\right\} \\
& =1-P\left\{\begin{array}{c}
\text { no transmission } \\
\text { in the vulnerable interval }
\end{array}\right\} \\
& =1-e^{-\lambda D_{\mathrm{msg}}} .
\end{aligned}
$$

If $\lambda D_{\mathrm{msg}} \ll 1$, then $p \simeq \lambda D_{\mathrm{msg}}=\lambda M / R_{\mathrm{b}}$ is $\quad \mathrm{a}$ valid approximation.

We now consider a rigorous detection-theoretic approach for the evaluation of the average link BER in the presence of INI and RESGO MAC protocol. We show that the widely used Gaussian assumption for the distribution of the interference noise has limited validity, especially in dense ad hoc wireless networks. In order to analytically characterize the impact of interference, we refer to a regular square-grid topology, as shown in Fig. 1, and we assume that the link of interest is placed at the center of the network. The applicability of the obtained results to a scenario with mobile nodes is justified by the constraint that nodes, while moving, are confined within the fixed network surface. We consider the BER performance in a scenario where only the nodes from first square tier around $n_{D}$, denoted as Tier 1, interfere with an ongoing link transmission. Obviously, this is not realistic, since all nodes in the network interfere. However, it can be shown that the interference originating from nodes in external tiers (beyond Tier 1) is negligible.

Considering the link between nodes $n_{S}$ and $n_{D}$ shown in Fig. 1, the nodes in Tier 1 can be classified in two groups of nodes. 
Group 1) The number of nodes in this group, which is denoted ${ }^{4}$ as nodes 11 , is equal to 3 . Each of them generates an interference power equal to $P_{\mathrm{r}}^{(11)}=P_{\mathrm{r}}=$ $\alpha P_{\mathrm{t}} / d_{\text {link }}^{2}$. The received bit energy is $E_{\mathrm{b}}^{(11)}=E_{\mathrm{b}}=$ $P_{\mathrm{r}} / R_{\mathrm{b}}$.

Group 2) The number of nodes in this group, denoted as nodes $_{12}$, is equal to 4 . Each of them generates an interference power equal to $P_{\mathrm{r}}^{(12)}=P_{\mathrm{r}} / 2=$ $\alpha P_{\mathrm{t}} / 2 d_{\text {link }}^{2}$. The received bit energy is $E_{\mathrm{b}}^{(12)}=$ $E_{\mathrm{b}} / 2$.

Averaging properly over the interfering nodes and assuming that BPSK is the modulation format, using combinatorics, it can be shown that the average link BER can be written as follows [19]:

$$
\begin{aligned}
\overline{\mathrm{BER}}_{\text {link }}= & \sum_{n_{11}=0}^{\text {nodes }_{11}} \sum_{n_{12}=0}^{\text {nodes }_{12}}\left(\begin{array}{c}
\operatorname{modes}_{11} \\
n_{11}
\end{array}\right)\left(\begin{array}{c}
\operatorname{modes}_{12} \\
n_{12}
\end{array}\right) p^{n_{11}+n_{12}} \\
& \cdot(1-p)^{\text {nodes }_{11}+\operatorname{nodes}_{12}-n_{11}-n_{12}} \frac{1}{2^{n_{11}+n_{12}}} \\
& \cdot \sum_{i_{11}=1}^{2^{n_{11}}} \sum_{i_{12}=1}^{2^{n_{12}}} Q\left[\frac { \sqrt { E _ { b } } } { \sigma } \left(1+\operatorname{term}_{11}\left(n_{11}, i_{11}\right)\right.\right. \\
& \left.\left.+\frac{\operatorname{term}_{12}\left(n_{12}, i_{12}\right)}{\sqrt{2}}\right)\right]
\end{aligned}
$$

where $\sigma=\sqrt{F k T_{0} / 2}, p=1-e^{-\lambda M / R_{\mathrm{b}}} \simeq \lambda M / R_{\mathrm{b}}$, and the notation term $\mathrm{i}_{\mathrm{i} j}(h, k)$ has the following meaning.

1) $\mathbf{i} \longrightarrow$ refers to the tier (up to this point, there is only Tier 1).

2) $j \longrightarrow$ refers to the $j$ th subgroup within the tier, containing nodes $\mathbf{i} j$ nodes.

3) $h=0, \ldots$, nodes $_{\mathbf{i} j} \longrightarrow$ indicates how many nodes from Group $\mathbf{i} j$ are transmitting.

4) $k=1, \ldots, 2^{h} \longrightarrow$ is an index of the possible ways in which $h$ nodes can transmit-since we are considering binary transmissions, the total number of ways in which the nodes can transmit is $2^{h}$.

The quantity $\operatorname{term}_{\mathbf{i} j}(h, k)$ will be used to characterize the overall interfering signal, by combining the signs of the component interfering signals. In particular, we define

$$
\begin{aligned}
& \operatorname{term}_{11}\left(0,2^{0}\right)=0 \\
& \operatorname{term}_{11}(1,1)=+1 \\
& \operatorname{term}_{11}\left(1,2^{1}\right)=-1 \\
& \operatorname{term}_{11}(2,1)=+1+1=+2 \\
& \operatorname{term}_{11}(2,2)=+1-1=0 \\
& \cdots \text { term }_{11}\left(2,2^{2}\right)=-1-1=-2 \\
& \operatorname{term}_{11}(3,1)=+1+1+1=+3 ; \ldots \\
& \cdots \text { term }_{11}\left(3,2^{3}\right)=-1-1-1=-3 \\
& \operatorname{term}_{12}(h, k)=\operatorname{term}_{11}(h, k) h=0, \ldots, 3 \quad k=1, \ldots, 2^{h} \\
& \operatorname{term}_{12}(4,1)=+1+1+1+1=+4 ; \ldots \\
& \cdots \operatorname{term}_{12}\left(4,2^{4}\right)=-1-1-1-1=-4 .
\end{aligned}
$$

\footnotetext{
${ }^{4}$ In the considered notation for a group of nodes, the first boldface number refers to the tier, whereas the second number refers to the particular subgroup in the corresponding tier.
}

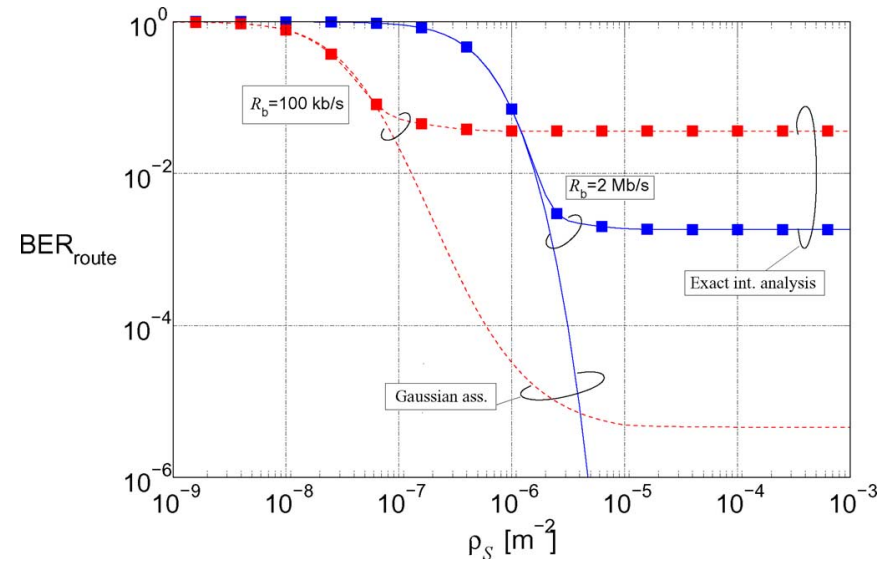

Fig. 2. Route BER as a function of the node spatial density, considering the interference originating only from nodes in Tier 1 . The main parameters are $P_{\mathrm{t}}=1 \mathrm{~mW}, G_{\mathrm{t}}=G_{\mathrm{r}}=f_{1}=1, \lambda=0.5 \mathrm{msg} / \mathrm{s}, M=1000 \mathrm{~b} / \mathrm{msg}$, $F=6 \mathrm{~dB}$, and $N=300$. Various values of the data rate $R_{b}$ are considered. For comparison, the route BER predicted by the Gaussian assumption is also shown.

The route BER (corresponding to the average link BER) can be written as ${ }^{5}$

$$
\mathrm{BER}_{\text {route }}=1-\left(1-\overline{\mathrm{BER}}_{\text {link }}\right)^{\bar{n}_{\mathrm{h}}} .
$$

The route BER performance, in a scenario with $P_{\mathrm{t}}=1 \mathrm{~mW}$, $G_{\mathrm{t}}=G_{\mathrm{r}}=f_{\mathrm{l}}=1, N=300, F=6 \mathrm{~dB}, \lambda=0.5 \mathrm{msg} / \mathrm{s}$, and $M=1000 \mathrm{~b} / \mathrm{msg}$, is shown in Fig. 2, for two values of the data rate $R_{\mathrm{b}}(2 \mathrm{Mb} / \mathrm{s}$ and $100 \mathrm{~kb} / \mathrm{s}$, respectively). In the figure, for the sake of comparison, the route BER under the Gaussian assumption for the interference noise is also shown-the details of the derivation of the route BER under the Gaussian assumption can be found in [16] and [17]. While the correct interference analysis predicts a very high BER floor, the route BER predicted with the Gaussian assumption presents a much lower BER floor. We observe, however, that for sufficiently low values of the node spatial density, i.e., for sufficiently high route BER values, the route BER predicted by the Gaussian assumption is a very good approximation for the route BER predicted with the exact interference analysis. This phenomenon can be given an intuitive explanation. For low values of the node spatial density, the interfering nodes are relatively distant from the receiver. Therefore, they interfere more or less in the same way at the receiver, and the presence of a sufficiently large number of nodes justifies the use of the central limit theorem (CLT) to characterize the distribution of the interfering noise as Gaussian [26]. However, as the node spatial density increases, the closest neighbors (in Tier 1) interfere much more (relatively) than the other nodes, and this prevents the application of the CLT, since the outer nodes will contribute a negligible amount of interference. Moreover, the presence of neighboring nodes interfering with approximately the same power of the received useful signal leads to an unavoidable BER floor, which is analytically characterized in the following paragraph.

\footnotetext{
${ }^{5}$ The route BER given by (9) is not exactly the average route BER but the route BER corresponding to the average link BER. Our results, however, show
} that the average route BER is slightly lower than the BER given by (9). 
Considering expression (9) for the route BER based on the exact interference analysis, it is possible to find an analytic expression for the BER floor. In fact, it is sufficient to isolate the $Q(\cdot)$ terms whose arguments are lower than or equal to zero to find the asymptotic (for large node spatial densities) route BER. Since, in these cases, $1<Q(\cdot) \leq 1 / 2$, it is sufficient to consider the "weight" of these $Q(\cdot)$ terms: the term with the highest weight will clearly be the dominant one. After a simple analysis, one finds that

$$
\lim _{\rho_{S} \rightarrow \infty} \overline{\mathrm{BER}}_{\text {link }} \simeq \frac{3 p}{4}=\frac{3\left(1-e^{-\frac{\lambda M}{R_{\mathrm{b}}}}\right)}{4} \simeq \frac{3 \lambda M}{4 R_{\mathrm{b}}}
$$

where the two approximations hold provided that $p \ll 1$, i.e., there is low traffic. Therefore, the route BER becomes

$$
\begin{aligned}
\mathrm{BER}_{\text {route,floor }}^{\text {RESGO }} & \triangleq \lim _{\rho_{S} \rightarrow \infty} \mathrm{BER}_{\text {route }} \\
& =1-\left(1-\lim _{\rho_{S} \rightarrow \infty} \overline{\mathrm{BER}}_{\text {link }}\right)^{\bar{n}_{\mathrm{h}}} \\
& \simeq 1-\left(1-\frac{3 p}{4}\right)^{\bar{n}_{\mathrm{h}}} \\
& \simeq \frac{3 \bar{n}_{\mathrm{h}} p}{4}=\frac{3 \bar{n}_{\mathrm{h}} \lambda M}{4 R_{\mathrm{b}}}
\end{aligned}
$$

where, in the last passage, we have considered a first order Taylor series expansion exploiting the fact that $p \ll 1$.

To verify our analysis, we also compute the route BER via Monte Carlo simulations. In the simulation, each node transmits packets according to RESGO MAC protocol. For each bit received by the receiver, the detection decision is made based on the total received signal (i.e., the information-bearing signal from the transmitter, the interference signals, and the thermal noise). If the received bit is different from the original bit sent by the transmitter, it is declared as a bit error. Through a Monte Carlo approach, the link BER can then be obtained by calculating the ratio between the number of bits received in error and the total number of bits received. Finally, the route BER can be obtained from the link BER using (9). In Fig. 3, we compare the route BER obtained from the analysis with that obtained by simulation. The parameters used are the same as those in Fig. 2. It can be observed that both analytical and simulation results are in good agreement.

As previously observed in Fig. 2, the route BER predicted by the exact interference analysis coincides with that predicted by the Gaussian assumption for BER values higher than that corresponding to the BER floor, an approximate expression for which is given by (11). In other words, the Gaussian assumption is valid for 1) low node spatial density and/or 2) low traffic intensity. However, in these conditions, the interference power is not the dominant source of noise. The failure of the Gaussian assumption in scenarios with medium/high node spatial density is due to the fact that there are a few nodes, especially in Tier 1, which interfere significantly more than the others with the transmitted signal. In particular, if a single node from Group 11 interferes destructively with the transmitted signal,

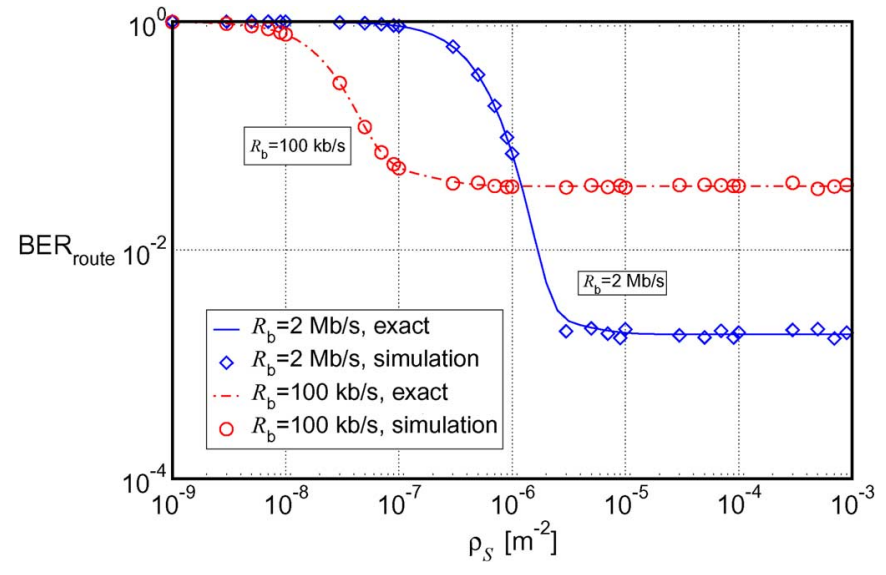

Fig. 3. Route BER as a function of the node spatial density, considering the interference originating only from nodes in Tier 1 . The main parameters are $P_{\mathrm{t}}=1 \mathrm{~mW}, G_{\mathrm{t}}=G_{\mathrm{r}}=f_{1}=1, \lambda=0.5 \mathrm{msg} / \mathrm{s}, M=1000 \mathrm{~b} / \mathrm{msg}$, $F=6 \mathrm{~dB}$, and $N=300$. Various values of the data rate $R_{b}$ are considered. Analytical and simulation route BER results are shown.

all hope is lost. These observations suggest the following simple approximation for the route BER:

$$
\begin{aligned}
\mathrm{BER}_{\text {route }} \simeq \max \left\{\left[1-\left(1-\mathrm{BER}_{\text {link }}^{\text {Gauss }}\right)^{\bar{n}_{\mathrm{h}}}\right], \frac{3 \bar{n}_{\mathrm{h}} p}{4}\right\} \\
=\max \left\{\left[1-\left[1-Q\left(\sqrt{\frac{2 P_{\mathrm{r}}}{P_{\text {thermal }}+P_{\text {int }}}}\right)\right]^{\bar{n}_{\mathrm{h}}}\right]\right. \\
\\
\left.\frac{3 \bar{n}_{\mathrm{h}} \lambda M}{4 R_{b}}\right\}
\end{aligned}
$$

where the expression for the link BER under the Gaussian assumption for the interference noise [i.e., $\mathrm{BER}_{\text {link }}^{\text {Gauss }}$ in the first term in the maximum operator in (12)] is derived in [16] and [17]. By adding the interference powers contributed from the interfering nodes in the concentric tiers in the square-grid topology in Fig. 1 and recalling that each of them has to be weighed by the same probability $p=1-e^{\lambda M / R_{\mathrm{b}}}$, it can be shown that the average interference power can be expressed as

$$
\begin{aligned}
P_{\mathrm{int}}^{\mathrm{RESGO}} & =\alpha P_{\mathrm{t}} \rho_{\mathrm{S}}\left(1-e^{-\frac{\lambda M}{R_{\mathrm{b}}}}\right) \Delta_{A}(N) \\
& \simeq \alpha P_{\mathrm{t}} \rho_{\mathrm{S}} \frac{\lambda M}{R_{\mathrm{b}}} \Delta_{A}(N)
\end{aligned}
$$

where the last approximation holds for low traffic load, and

$$
\Delta_{A}(N) \triangleq\left[\sum_{i=1}^{\lfloor\sqrt{N} / 2\rceil}\left(\frac{6}{i^{2}}+8 \sum_{j=1}^{i-1} \frac{1}{i^{2}+j^{2}}\right)-1\right] \text {. }
$$

The quantity $\Delta_{A}(N)$ depends on the geometry of the node distribution (square grid). Preliminary results suggest, however, that the difference between various regular topologies is negligible. It is possible to show that there is excellent agreement between the exact route BER and the approximate route BER for the considered network scenarios. 


\section{B. Reserve-Listen-and-Go (RESLIGO) MAC Protocol}

Unlike the case with RESGO MAC protocol considered above, in the case with the second MAC protocol ${ }^{6}$ proposed in [16], [17], and [19], a source node, after reserving a multihop communication route to its destination, first senses the shared radio channel and then, if the channel is idle, starts transmitting. For this reason, we refer to this MAC protocol as RESLIGO. If the source node senses that another multihop route is active, it delays the activation of its reserved multihop route. It is possible to show that, in an ad hoc wireless network 1) with a number of nodes not extremely large and 2) with sufficiently high receiver sensitivity (so that a node can "hear" any other node in the network), the use of this MAC protocol is such that only one route at a time is active. More details on the impact of the receiver sensitivity on the performance of ad hoc wireless networks can be found in [29]. In this way, the interference between nodes is basically reduced to zero, and the corresponding performance, in terms of BER, is equal to that in the ideal case. In particular, all the results, shown in Section VI, relative to an ideal networkcommunication scenario, are valid also in a realistic networkcommunication scenario where RESLIGO MAC protocol is in use.

\section{Performance Analysis of RESGO MAC Protocol in a Scenario With Node Mobility}

Provided that the maximum tolerable BER at the end of a multihop route is higher than $\mathrm{BER}_{\text {route, floor }}^{\mathrm{RESGO}}$ and, consequently, the Gaussian assumption for the interference noise can be applied, the link SNR can be written as follows:

$$
\mathrm{SNR}_{\text {link }}^{\text {int }}=\frac{E_{\text {bit }}}{E_{\text {thermal }}+E_{\text {int }}^{\text {RESGO }}}
$$

where $E_{\text {int }}^{\mathrm{RESGO}}$ is the interference energy. Assuming that the sum of the interfering signals can be considered as an additive white $^{7}$ process, it follows that $E_{\text {int }}^{\mathrm{RESGO}}=P_{\text {int }}^{\mathrm{RESGO}} / B$, where $P_{\text {int }}^{\mathrm{RESGO}}$ is the received interference power, and $B$ is the transmission bandwidth. The expression for $P_{\text {int }}$ is given by (13) — in general, the interference power depends on the specific MAC protocol in use and the spatial distribution of the nodes.

In Section VI, the impact of the INI (i.e., interference), when RESGO MAC protocol is used, will be evaluated as follows.

1) First, the impact of the INI will be evaluated simply by substituting in the link SNR expression (15) the interference noise power given by (13). Then, the route BER will be evaluated through the novel semi-analytical approach (which takes into account the node mobility) described in Section V. We will refer to this BER as BER Gauss This is obviously not rigorous, since, because of mobility, the node topology is likely not to be regular (square

\footnotetext{
${ }^{6}$ In [16] and [17], this MAC protocol was incorrectly referred to as per-route carrier sense multiple access (PR-CSMA), since it resembles CSMA [27], [28], in the sense that, before activating the reserved route, a source node senses the channel and transmits only if the channel is idle.

${ }^{7}$ The assumption of white interference noise is reasonable in a scenario with antipodal modulation (e.g., BPSK) and asynchronism between transmissions from different nodes. In fact, in this case, it is very likely that any shifted version of the interfering signal is highly uncorrelated with the original version.
}

grid), so that the considered expression for the average interference power is not extremely accurate. However, owing to the assumption that the nodes cannot exit the network surface and due to the focus of this paper (the analysis of the impact of node mobility), we will use the simple closed-form expression (13) to take into account the interference. A more rigorous analysis, taking explicitly into account the effective node distribution and based on computer simulations, can be carried out following the approach proposed in [30] and [31].

2) At this point, one should compare the route BER computed as described in the previous item (under the Gaussian assumption) with the BER floor due to RESGO MAC protocol, and select the maximum one. The extension of the analysis carried out in Section III-A, valid for static nodes placed at the vertices of a square grid, in a scenario with node mobility is difficult and requires heavy use of simulation. In Section III-A, (11) for the route BER floor with RESGO MAC protocol has been found. From this equation, one can conclude that the route BER floor with RESGO MAC protocol depends only on the traffic load $\lambda M$ and the data rate $R_{b}$, but not, for example, on the node spatial density. Motivated by this observation, and since in the current scenario with mobility we are assuming that nodes cannot move out of the network surface, we still assume that the route BER has the same expression given by (11). In fact, under the assumption that nodes do not move out of the network surface, each node experiences, on average, the same level of interference of a static scenario.

\section{SWITCHING MODELS}

\section{A. Opportunistic Nonreservation-Based Switching (ONRBS)}

Under this switching model, a source node, in need of communicating with a destination node, does not statically reserve intermediate relay nodes. Instead, once the transmission from the source node has started, consecutive links, from source to destination, are chosen opportunistically, based on their lengths. In other words, at the moment of route creation, ${ }^{8}$ a "tentative" multihop route (or, possibly, more than a single multihop route [18]) from source to destination is created. Afterwards, there is adaptive maintenance of the route links. More precisely, if two consecutive nodes (constituting a link) of the originally created route move too far from each other, the starting node of this link will choose another node at average distance $\Theta\left(1 / \sqrt{\rho_{\mathrm{S}}}\right)$. As underlined before, the assumptions that nodes 1) are confined within the network surface with fixed area and 2) are mobile imply that, on average, a node can always find a neighboring node at distance $\Theta\left(1 / \sqrt{\rho_{\mathrm{S}}}\right)$. Note that it might happen that a neighboring node at distance $\Theta\left(1 / \sqrt{\rho_{\mathrm{S}}}\right)$ is not immediately present: in this sense, the overall transmission from source to destination might incur an additional delay,

\footnotetext{
${ }^{8}$ As mentioned in the general assumptions considered in Section II, the phase of route creation is not considered explicitly since it is beyond the scope of this paper. However, we note that it should depend on the broadcast sent by a source node. The route discovery process can be studied according to the principles of broadcast percolation [32].
} 
which, however, is not considered in the analysis presented in this paper. Moreover, opportunistic link creation/activation from source to destination might require a significant exchange of control messages among the intermediate nodes of the multihop route, reducing the overall transfer of "useful" information. This is the price one has to pay for increased robustness against mobility, as will be shown in Section VI. Further research is needed to address these important aspects of ONRBS-based ad hoc wireless network communications. In this paper, for the sake of simplicity, we assume a "perfect" control channel mechanism for route maintenance: in particular, we assume that a neighboring node at distance $\Theta\left(1 / \sqrt{\rho_{\mathrm{S}}}\right)$ can be "immediately" found for the activation of the next link.

\section{B. Reservation-Based Switching (RBS)}

In this case, during the route discovery process, intermediate relay nodes are reserved in a static way. In other words, once a route is created, the order of the intermediate relay nodes does not change for the entire duration of the transmission, regardless of the actual lengths of the links. Obviously, for a sufficiently high node mobility level, even assuming that the initial setup of a multihop route is characterized by a sequence of hops with length $\Theta\left(1 / \sqrt{\rho_{\mathrm{S}}}\right)$, the lengths of the latest links of the route could significantly change with respect to their initial lengths. Hence, we expect that the robustness of RBS to node mobility is significantly reduced with respect to that of ONRBS. This degradation should be limited, however, if the mobility pattern is characterized by frequent changes of direction (such mobility model is described in Section V-B. We also note that the source node or intermediate nodes could have to wait in order to find a neighbor at distance $\Theta\left(1 / \sqrt{\rho_{\mathrm{S}}}\right)$. Consequently, the source could be getting away from the destination during this time. Since we are interested in a steady-state networkcommunication regime and a delay analysis is not the focus of this paper (where the focus is on an average BER performance analysis), we do not explicitly consider this aspect in the remainder of this paper.

\section{Mobility Models}

The mobility status of a node can be described in terms of its speed, which is denoted as $v$, and its direction angle (with respect to a horizontal axis), which is denoted as $\theta$. Two possible mobility models are considered in the following, but we underline that the proposed approach is applicable to other cases, provided that a statistical description of nodes' mobility model is available.

\section{A. DP Mobility Model}

In this case, during the transmission of a message, the direction and speed of the two nodes at the ends of a link are constant. Based on this assumption, we now investigate how this mobility model can be combined with the two considered switching strategies.

1) ONRBS: Consecutive links are considered "independent" of each other. More precisely, the mobility status of a node during a message transmission in a link (where such node
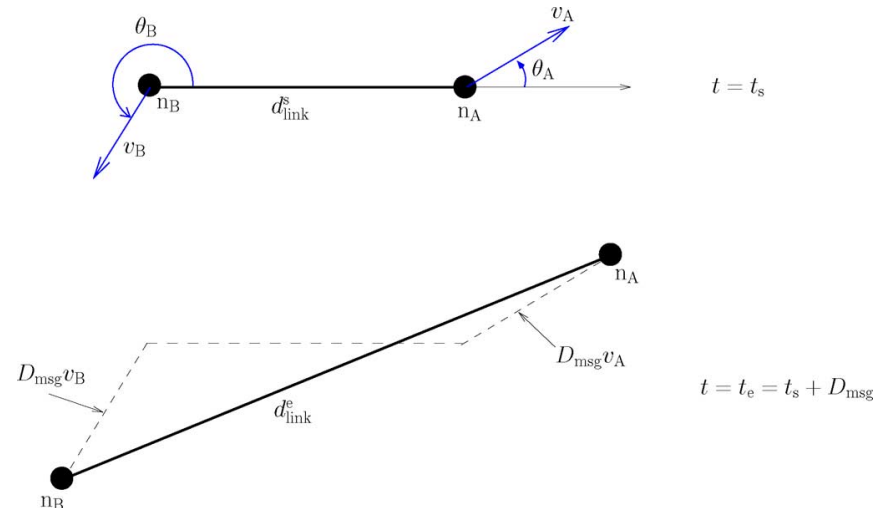

Fig. 4. Link evolution during a message transmission in the case of DP mobility model.

is the final node) will be independent of its mobility status during the message transmission in the consecutive link (where such node is the initial node).

Under the assumption of a DP mobility model, we now outline the evolution, during a message transmission, of an intermediate link of a multihop route. We refer to the two nodes of a link as $n_{A}$ and $n_{B}$. We assume that these nodes have constant speeds and direction angles, which are denoted as $\left(v_{\mathrm{A}}, \theta_{\mathrm{A}}\right)$ and $\left(v_{\mathrm{B}}, \theta_{\mathrm{B}}\right.$, respectively), during the transmission of a message. Their initial distance is $d_{\text {link }}$. The link statuses at the activation $\left(t=t_{\mathrm{s}}\right)$ and at the end $\left(t=t_{\mathrm{e}}=t_{\mathrm{s}}+D_{\mathrm{msg}}\right)$ of a message transmission are shown in Fig. 4. Simple geometric considerations allow one to express the final link length $d_{\text {link }}^{\mathrm{e}}$ as

$$
\begin{aligned}
d_{\text {link }}^{\mathrm{e}}=\left\{d_{\text {link }}^{2}\right. & +D_{\mathrm{msg}}^{2}\left(v_{\mathrm{A}}^{2}+v_{\mathrm{B}}^{2}\right)-2 v_{\mathrm{A}} v_{\mathrm{B}} D_{\mathrm{msg}}^{2} \cos \left(\theta_{\mathrm{A}}-\theta_{\mathrm{B}}\right) \\
& \left.+2 d_{\text {link }} D_{\mathrm{msg}}\left(v_{\mathrm{A}} \cos \theta_{\mathrm{A}}-v_{\mathrm{B}} \cos \theta_{\mathrm{B}}\right)\right\}^{0.5}
\end{aligned}
$$

In order to make an average performance analysis based on the framework developed in Section II, it is expedient to compute an average link length denoted as $\bar{d}_{\text {link }}$. As a simple and meaningful average link length, we consider the arithmetic mean between $d_{\text {link }}^{s}=d_{\text {link }}$ and $d_{\text {link }}^{\text {e }}$, i.e.,

$$
\begin{aligned}
\bar{d}_{\text {link }} \triangleq \frac{d_{\text {link }}^{s}+d_{\text {link }}^{\mathrm{e}}}{2} & \\
=\frac{d_{\text {link }}}{2}+\frac{1}{2}\{ & d_{\text {link }}^{2}+D_{\text {msg }}^{2}\left(v_{\mathrm{A}}^{2}+v_{\mathrm{B}}^{2}\right)-2 v_{\mathrm{A}} v_{\mathrm{B}} \\
& \cdot D_{\mathrm{msg}}^{2} \cos \left(\theta_{\mathrm{A}}-\theta_{\mathrm{B}}\right)+2 d_{\text {link }} D_{\mathrm{msg}} \\
& \left.\times\left(v_{\mathrm{A}} \cos \theta_{\mathrm{A}}-v_{\mathrm{B}} \cos \theta_{\mathrm{B}}\right)\right\}^{0.5}
\end{aligned}
$$

At this point, we assume that the average link SNR during the transmission of a message can be obtained from (6), provided that $d_{\text {link }}$ is replaced by $\bar{d}_{\text {link }}$. The fundamental difference with the case of a static topology is the fact that $\bar{d}_{\text {link }}$ is a random variable, whose realization depends on the realizations of $\left(v_{\mathrm{A}}, \theta_{\mathrm{A}}\right)$ and $\left(v_{\mathrm{B}}, \theta_{\mathrm{B}}\right)$.

In general, a Monte Carlo simulation-based approach for the evaluation of the BER at the end of a multihop route with an average number of hops can be considered. For the sake of simplicity, the mobility patterns of different nodes are assumed to be independent. We define as $\zeta_{i} \triangleq\left(v_{i}, \theta_{i}, v_{i+1}, \theta_{i+1}\right)$ the ensemble of speed and movement angle realizations of the two 
nodes constituting the $i$ th hop, $i \in\left\{1, \ldots, \bar{n}_{\mathrm{h}}\right\}$, during a message transmission. ${ }^{9}$ Clearly, $\bar{d}_{\text {link }}=\bar{d}_{\text {link }}\left(\zeta_{i}\right)$. We denote the $i$ th link BER (under the Gaussian assumption for the interference noise), corresponding to the realization $\zeta_{i}$, as $\operatorname{BER}_{\text {link }}^{\text {Gauss }}\left(\zeta_{i}\right)$. Hence, the final BER at the end of a multihop route with an average number of hops (under the Gaussian assumption for the interference noise) corresponding to an overall realization ensemble, ${ }^{10}$ denoted as $\zeta_{\text {ONRBS }} \triangleq\left(\zeta_{1}, \ldots, \zeta_{\bar{n}_{\mathrm{h}}}\right)$, can be written as

$$
\operatorname{BER}_{\text {route }}^{\text {Gauss }}\left(\zeta_{\text {ONRBS }}\right)=1-\prod_{i=1}^{\bar{n}_{\mathrm{h}}}\left[1-\operatorname{BER}_{\text {link }}^{\text {Gauss }}\left(\zeta_{i}\right)\right]
$$

Considering a sufficiently large number $\eta$ of realization ensembles, i.e., $\zeta_{\text {ONRBS }}^{(j)}=\left(\zeta_{1}^{(j)}, \ldots, \ldots, \zeta_{\bar{n}_{\mathrm{h}}}^{(j)}\right), j \in\{1, \ldots, \eta\}$, the average BER under the Gaussian assumption can be estimated as

$$
\mathrm{BER}_{\text {route }}^{\text {Gauss }}=\frac{\sum_{j=1}^{\eta} \mathrm{BER}_{\text {route }}\left(\zeta_{\text {ONRBS }}^{(j)}\right)}{\eta} .
$$

Therefore, owing to the assumption that the route BER floor is given by (11), the route BER can be finally expressed as follows:

$$
\mathrm{BER}_{\text {route }}=\max \left\{\mathrm{BER}_{\text {route }}^{\text {Gauss }}, \bar{n}_{\mathrm{h}} \frac{3 \lambda M}{4 R_{b}}\right\} .
$$

2) RBS: Once a multihop route has been established, a message flows through the originally reserved links of the route, regardless of their evolution (due to nodes' movement). In other words, when a message reaches an intermediate link of the route, the message transmission over this link is activated regardless of the corresponding link length. This situation is depicted in Fig. 5 (in the figure, $\bar{n}_{\mathrm{h}}=6$ ), where it is assumed that the mobility status of each node remains constant for the entire message transmission along the activated route. Note that a fundamental assumption, which is implicit in Fig. 5, is the fact that a message, before being retransmitted by an intermediate node, needs to be completely received. In other words, an intermediate node cannot start forwarding a message before having received it completely. This is consistent with a realistic communication scheme where complete regeneration is considered at each intermediate node. However, the use of particular coding/decoding techniques could allow retransmission before complete reception. This aspect is beyond the scope

\footnotetext{
${ }^{9}$ Note that $\left(v_{\mathrm{B}}^{(i)}, \theta_{\mathrm{B}}^{(i)}\right)$ and $\left(v_{\mathrm{A}}^{(i+1)}, \theta_{\mathrm{A}}^{(i+1)}\right)$ are the two consecutive mobility statuses relative to the same node. In the case of ONRBS, we assume that they are independent. This implies that the same node is associated with two different mobility statuses in a link where it is the final node and in the following link, where it is the starting node. In all cases, the number of hops is likely to be $\bar{\Theta}(\sqrt{N})$.

${ }^{10}$ Note that, due to ONRBS, it might happen that the number of hops between source and destination is actually larger than $\bar{n}_{\mathrm{h}}$. However, in order to make a simple and meaningful comparison with the case of RBS, we assume that the total number of hops from source to destination is still, on average, $\bar{n}_{\mathrm{h}}$.
}
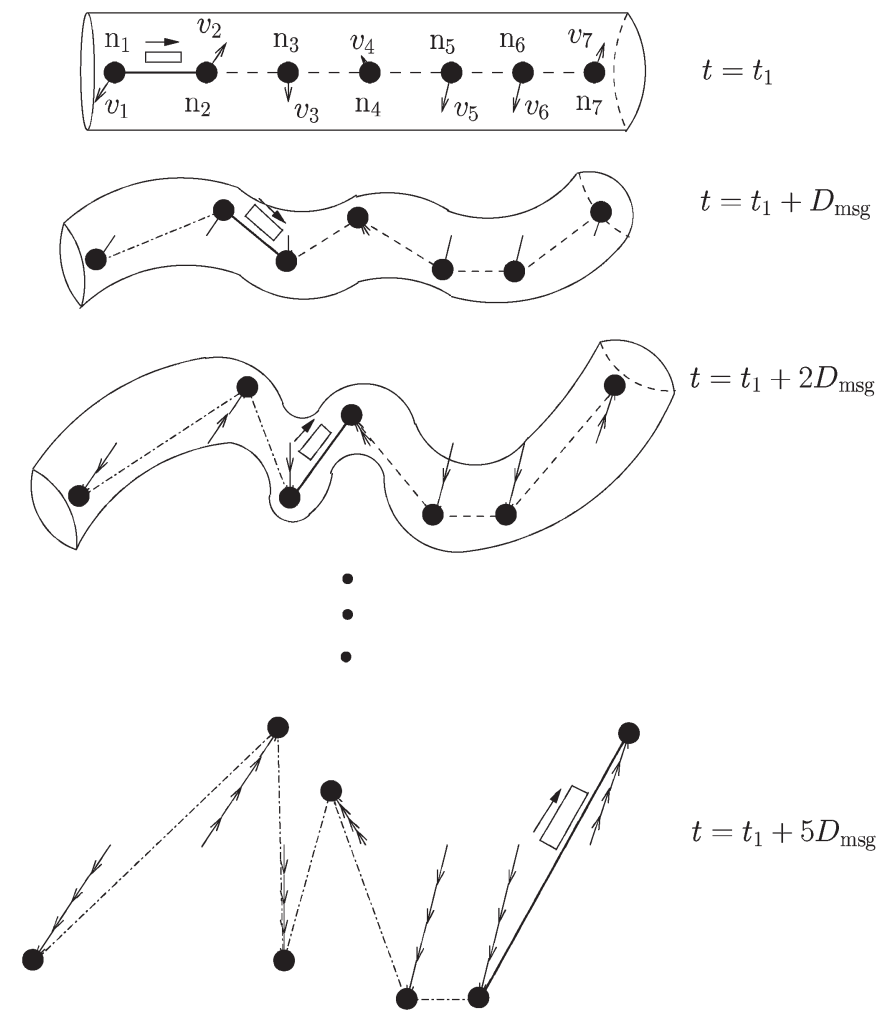

Fig. 5. Route evolution during a message transmission in the case of RBS.

of this paper, but we point out that the proposed approach could be extended to take this aspect into account.

It is intuitive to visualize each communication route as a "tube," inside which the messages generated at the source node flow to the destination node, at the end of the tube. As shown in Fig. 5 for a particular sequence of nodes' mobility statuses, while a message flows along the route, the corresponding tube bends (due to node mobility). In correspondence to the activation of the last link of the route in Fig. 5 (i.e, for $t=t_{1}+5 D_{\mathrm{msg}}$ ), no tube is shown, since it is immediate to realize that the tube "bent" too much in some regions (e.g., around node $n_{3}$ ). In other words, the tube is most probably going to break and needs to be "updated" (e.g., node $\mathrm{n}_{2}$ could communicate directly to node $\mathrm{n}_{4}$, bypassing node $\mathrm{n}_{3}$ ). In a realistic scenario, this updating would be carried out by the routing protocol. Intuitively, it is not difficult to realize that the higher the speed, the sooner a tube is going to break.

The approach considered in Section V-A1, for the evaluation of the BER in a network-communication scenario with ONRBS, can be extended to the case with RBS. In fact, for each link of the route, it is possible to compute the associated starting and final lengths, thus obtaining the average link length (as the arithmetic mean of starting and final lengths). For the first link, activated at $t=t_{1}$, the starting and final link lengths are

$$
\begin{aligned}
d_{\text {link }}^{(1, \mathrm{~s})}= & d_{\text {link }} \\
d_{\text {link }}^{(1, \mathrm{e})}=\{ & d_{\text {link }}^{2}+D_{\mathrm{msg}}^{2}\left(v_{1}^{2}+v_{2}^{2}\right)-2 v_{1} v_{2} D_{\mathrm{msg}}^{2} \cos \left(\theta_{1}-\theta_{2}\right) \\
& \left.\quad+2 d_{\text {link }} D_{\mathrm{msg}}\left(v_{1} \cos \theta_{1}-v_{2} \cos \theta_{2}\right)\right\}^{0.5}
\end{aligned}
$$


In general, for the $i$ th route link, between nodes $\mathrm{n}_{i}$ and $\mathrm{n}_{i+1}$, activated at time instant $t=t_{1}+(i-1) D_{\mathrm{msg}}$, the starting and ending link lengths can be written as follows, ${ }^{11}$ respectively:

$$
\begin{aligned}
& d_{\text {link }}^{(i, \mathrm{~s})}=\left\{d_{\mathrm{link}}^{2}+\left[(i-1) D_{\mathrm{msg}}\right]^{2}\left(v_{i}^{2}+v_{i+1}^{2}\right)\right. \\
& -2 v_{i} v_{i+1}\left[(i-1) D_{\mathrm{msg}}\right]^{2} \cos \left(\theta_{i}-\theta_{i+1}\right) \\
& \left.+2 d_{\text {link }}(i-1) D_{\mathrm{msg}}\left(v_{i} \cos \theta_{i}-v_{i+1} \cos \theta_{i+1}\right)\right\}^{0.5} \\
& d_{\text {link }}^{(i, \mathrm{e})}=\left\{d_{\text {link }}^{2}+\left(i D_{\mathrm{msg}}\right)^{2}\left(v_{i}^{2}+v_{i+1}^{2}\right)\right. \\
& -2 v_{i} v_{i+1}\left(i D_{\mathrm{msg}}\right)^{2} \cos \left(\theta_{i}-\theta_{i+1}\right) \\
& \left.+2 d_{\text {link }} i D_{\mathrm{msg}}\left(v_{i} \cos \theta_{i}-v_{i+1} \cos \theta_{i+1}\right)\right\}^{0.5} \text {. }
\end{aligned}
$$

The average length of the $i$ th link, which is denoted as $\bar{d}_{\text {link }}^{(i)}$, can simply be obtained by considering the arithmetic mean between the starting link length and the ending link length, i.e., $\bar{d}_{\text {link }}^{(i)}=\left(d_{\text {link }}^{(i, \mathrm{~s})}+d_{\text {link }}^{(i, \mathrm{e})}\right) / 2$. The BER at the end of the $i$ th link under the Gaussian assumption for the interference noise, denoted as $\mathrm{BER}_{\operatorname{link} i}^{\mathrm{Gauss}}$, is obtained by substituting $d_{\text {link }}$ in (6) for $\bar{d}_{\text {link }}^{(i)}$, which depends on $\left(v_{i}, \theta_{i}, v_{i+1}, \theta_{i+1}\right)$. In this case, the sequence of average link lengths $\left\{\bar{d}_{\text {link }}^{(1)}, \ldots, \bar{d}_{\text {link }}^{\left(\bar{n}_{\mathrm{h}}\right)}\right\}$ depends on the ensemble of the realizations of the nodes' mobility statuses, which we define as $\zeta_{\mathrm{RBS}} \triangleq\left\{\left(v_{1}, \theta_{1}\right), \ldots,\left(v_{\bar{n}_{\mathrm{h}}}, \theta_{\bar{n}_{\mathrm{h}}}\right)\right\}$. The BER (under the Gaussian assumption for the interference noise) at the end of a multihop route described by the ensemble realization $\zeta_{\mathrm{RBS}}$ can then be written as

$\operatorname{BER}_{\text {route }}^{\text {Gauss }}\left(\zeta_{\text {RBS }}\right)=1-\prod_{i=1}^{\bar{n}_{\mathrm{h}}-1}\left[1-\operatorname{BER}_{\text {link } i}^{\text {Gauss }}\left(v_{i}, \theta_{i}, v_{i+1}, \theta_{i+1}\right)\right]$.

Considering, as in Section V-A1, a sufficiently large number $\eta$ of realization ensembles $\zeta_{\mathrm{RBS}}^{(j)}=\left\{\left(v_{1}^{(j)}, \theta_{1}^{(j)}\right),\left(v_{2}^{(j)}, \theta_{2}^{(j)}\right), \ldots\right.$, $\left.\left(v_{\bar{n}_{\mathrm{h}}}^{(j)}, \theta_{\bar{n}_{\mathrm{h}}}^{(j)}\right)\right\}, j \in\{1, \ldots, \eta\}$, the BER at the end of a multihop route with an average number of hops can be estimated as

$$
\mathrm{BER}_{\text {route }}^{\text {Gauss }}=\frac{\sum_{j=1}^{\eta} \mathrm{BER}_{\text {route }}^{\text {Gauss }}\left(\zeta_{\mathrm{RBS}}^{(j)}\right)}{\eta} .
$$

As at the end of the previous section, the final expression for the route BER is given by (19).

\section{B. DNP Mobility Model}

Unlike a network-communication scenario characterized by a DP mobility model, in a network scenario characterized by a DNP mobility model, a node can change the direction of movement during a message transmission. In particular, we break the message duration into a finite number $\Sigma$ of subintervals

\footnotetext{
${ }^{11}$ Note that this analysis makes the implicit assumption that the propagation time between two neighboring nodes is negligible compared to the duration of a message transmission. This is verified in all the considered cases, and it is reasonable to expect that it will be verified in almost any practical ad hoc wireless network where successive hops are made between neighboring nodes.
}

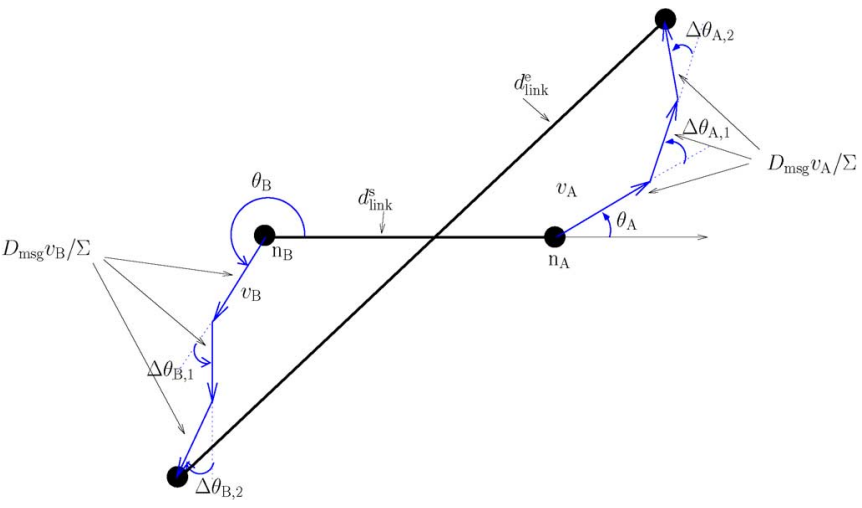

Fig. 6. Link evolution under the assumption of DNP mobility model. In particular, within a message transmission, $\Sigma=3$ subintervals, corresponding to different movement directions, are considered.

(or slots) of equal duration. Neglecting the propagation time, in each slot, a node, moving at speed $v$, covers a distance equal to $D_{\mathrm{msg}} v / \Sigma$. While we still assume that the speed of a node remains constant for the entire message duration, we assume that the movement angle can change from slot to slot. In particular, the change of angle $\Delta \theta$ at the end of a slot can be considered as a function of the speed: intuitively, the faster a node is moving, the smaller the change of direction can be-A more specific model will be introduced in Section VI. In Fig. 6, a pictorial example of the evolution of the link between two neighboring nodes (in the case with $\Sigma=3$ slots per message duration) is shown. The extension of the BER performance analysis to the current mobility model is outlined in the following.

1) ONRBS: In this case, we simply assume that the average link length $\bar{d}_{\text {link }}$ during a message transmission is obtained as the arithmetic mean of the average link lengths over consecutive slots. Since the average link length in the $j$ th slot, denoted as $\bar{d}_{\text {link } j}$, is

$$
\bar{d}_{\text {link } j}=\frac{d_{\text {link } j}^{s}+d_{\text {link } j}^{\mathrm{e}}}{2} \quad j=1, \ldots, \Sigma
$$

the average link length during a message transmission can thus be written as

$$
\bar{d}_{\text {link }}=\frac{\sum_{j=1}^{\Sigma} \bar{d}_{\text {link } j}}{\Sigma} .
$$

Denoting by $\mathrm{n}_{\mathrm{A}}$ and $\mathrm{n}_{\mathrm{B}}$ the two nodes at the ends of a link and defining by $\boldsymbol{\Delta} \boldsymbol{\theta}_{\mathrm{A}} \triangleq\left(\Delta \theta_{\mathrm{A}, 1}, \ldots, \Delta \theta_{\mathrm{A}, \Sigma-1}\right)$ and $\boldsymbol{\Delta} \boldsymbol{\theta}_{\mathrm{B}} \triangleq$ $\left(\Delta \theta_{\mathrm{B}, 1}, \ldots, \Delta \theta_{\mathrm{B}, \Sigma-1}\right)$ the sequences of direction changes in the $\Sigma$ consecutive time slots (within a message transmission) for node $n_{A}$ and node $n_{B}$, respectively, it is not difficult to conclude that the average link length $\bar{d}_{\text {link }}$ depends on $\left(v_{\mathrm{A}}, \theta_{\mathrm{A}}, \boldsymbol{\Delta} \boldsymbol{\theta}_{\mathrm{A}}\right)$ and $\left(v_{\mathrm{B}}, \theta_{\mathrm{B}}, \boldsymbol{\Delta} \boldsymbol{\theta}_{\mathrm{B}}\right)$.

As in the case of DP mobility model, in this case as well, a Monte Carlo simulation-based approach for the evaluation of the BER at the end of a multihop route with an average number of links can be used. A generic realization of the mobility statuses of the two nodes constituting the $i$ th link 
of the multihop route $\left(i \in\left\{1, \ldots, \bar{n}_{\mathrm{h}}\right\}\right)$, defined as $\zeta_{i}^{\prime}$, can be expressed as follows:

$$
\zeta_{i}^{\prime} \triangleq\left(v_{i}, \theta_{i}, \boldsymbol{\Delta} \boldsymbol{\theta}_{i}, v_{i+1}, \theta_{i+1}, \boldsymbol{\Delta} \boldsymbol{\theta}_{i+1}\right) .
$$

An ensemble realization of nodes' mobility statuses characterizing an entire multihop route is then denoted as $\zeta_{\text {ONRBS }}^{\prime} \triangleq$ $\left(\zeta_{1}^{\prime}, \ldots, \zeta_{\bar{n}_{\mathrm{h}}}^{\prime}\right)$. Given the link lengths, over a multihop route, associated with the extended realizations $\left\{\zeta_{i}^{\prime}\right\}$, the corresponding BER (under the Gaussian assumption for the interference noise distribution) at the end of the route can be computed. Considering a sufficiently large number $\eta$ of ensemble realizations $\left\{\zeta_{\mathrm{ONRBS}}^{\prime,(j)}\right\}_{j=1}^{\eta}$, it is possible to compute the average BER (under the Gaussian assumption) through Monte Carlo simulations, and the final expression for the route BER is obtained through (19).

2) RBS: Reasoning as in the case of ONRBS, it is possible to extend the approach considered for RBS in the case with a DP mobility model to a scenario characterized by a DNP mobility model. The average link length $\bar{d}_{i}$ of the $i$ th hop of a multihop route depends on $\zeta_{i}^{\prime} \triangleq\left(v_{i}, \theta_{i}, \boldsymbol{\Delta} \boldsymbol{\theta}_{i}^{\mathrm{RBS}}, v_{i+1}\right.$, $\left.\theta_{i+1}, \boldsymbol{\Delta} \boldsymbol{\theta}_{i+1}^{\mathrm{RBS}}\right)$. In this case, in order to describe the mobility statuses of the nodes of a multihop route, an ensemble realization is given by the sequence of mobility status realizations for all nodes, i.e.,

$$
\zeta_{\mathrm{RBS}}^{\prime} \triangleq\left(v_{1}, \theta_{1}, \boldsymbol{\Delta} \boldsymbol{\theta}_{1}^{\mathrm{RBS}}, \ldots, v_{\bar{n}_{\mathrm{h}}}, \theta_{\bar{n}_{\mathrm{h}}}, \boldsymbol{\Delta} \boldsymbol{\theta}_{\bar{n}_{\mathrm{h}}}^{\mathrm{RBS}}\right)
$$

where, unlike the case with ONRBS, the vector $\Delta \boldsymbol{\theta}_{i}^{\mathrm{RBS}}$ containing the angular direction changes for the $i$ th node is

$$
\begin{aligned}
\Delta \boldsymbol{\theta}_{i}^{\mathrm{RBS}} \triangleq\left(\Delta \theta_{i, 1}, \ldots, \Delta \theta_{i, \Sigma-1}, \ldots\right. \\
\left.\cdots \Delta \theta_{i,(i-1)(\Sigma-1)+1}, \ldots, \Delta \theta_{i, i(\Sigma-1)}\right) .
\end{aligned}
$$

Unlike the case with ONRBS, consecutive link realizations $\left(\zeta_{i}^{\prime}\right.$ and $\left.\zeta_{i+1}^{\prime}\right)$ are not independent. In other words, the ensemble realization $\zeta_{\mathrm{RBS}}^{\prime}$ depends on the single nodes' mobility realizations, rather than on the realizations of consecutive independent links. Finally, as in the case of DP mobility model, the BER at the end of a multihop route (under the Gaussian assumption for the interference noise), corresponding to the "overall" realization $\zeta_{\mathrm{RBS}}^{\prime}$, can be written as

$$
\begin{aligned}
& \operatorname{BER}_{\text {route }}^{\text {Gauss }}\left(\zeta_{\mathrm{RBS}}^{\prime}\right) \\
& =1-\prod_{i=1}^{\bar{n}_{\mathrm{h}}-1}\left[1-\mathrm{BER}_{\operatorname{link} i}^{\mathrm{Gauss}}\left(v_{i}, \theta_{i}, \boldsymbol{\Delta} \boldsymbol{\theta}_{i}^{\mathrm{RBS}}, v_{i+1}, \theta_{i+1}, \boldsymbol{\Delta} \boldsymbol{\theta}_{i+1}^{\mathrm{RBS}}\right)\right] .
\end{aligned}
$$

The final expression for the route BER can then be obtained as described in the previous sections.

\section{NumericAl RESUlts}

In this section, we analyze the impact of mobility, based on the semi-analytical approach previously introduced, in various ad hoc wireless networking scenarios, corresponding to different switching and mobility assumptions. The results are pre-

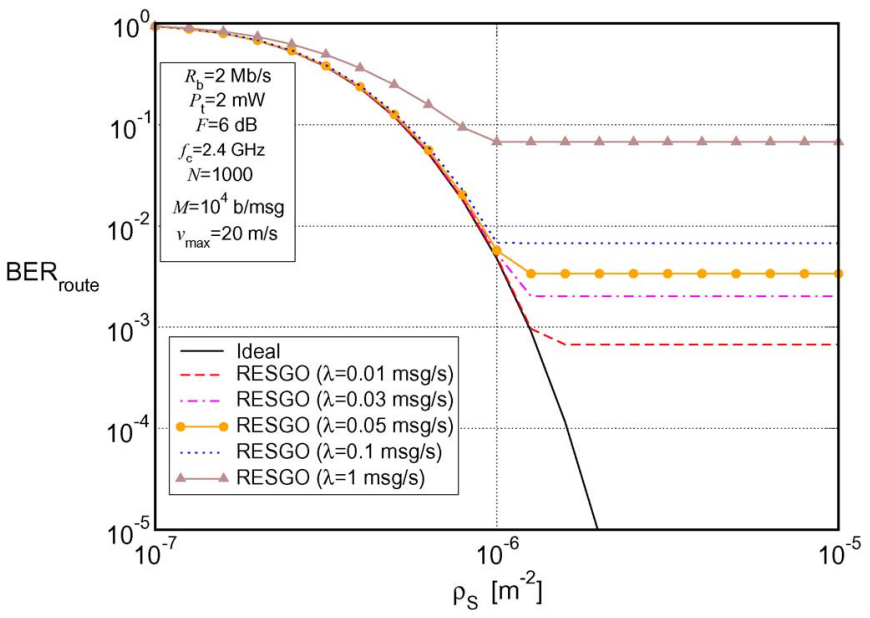

Fig. 7. Route BER versus node spatial density in the case of DP mobility model and ONRBS. The maximum node speed is $v_{\max }=20 \mathrm{~m} / \mathrm{s}$. The ideal (no INI) case and the realistic (INI) case with RESGO MAC protocol (for various values of the average traffic load $\lambda$ ) are considered.

sented according to the considered mobility model, either DP or DNP. In both cases, in order to evaluate the BER through the proposed semi-analytical Monte Carlo technique, a sufficiently large number $\eta$ of independent route realizations is considered. The major network parameter values used are indicated in the figures. In particular, the transmit power $\left(P_{\mathrm{t}}=2 \mathrm{~mW}\right)$ can be considered as typical of wireless sensor networks [33]. The proposed approach can be straightforwardly extended, as shown in [17], to the case of wireless local area networks $\left(P_{\mathrm{t}}=0.5 \mathrm{~W}\right)$ [34], [35] and smart dustlike networks $\left(P_{\mathrm{t}} \approx 1 \mu \mathrm{W}\right)$ [36]. The results presented hereafter hold, trendwise, also in the two latter scenarios.

\section{A. DP Mobility Model}

In this case, consecutive links are independent. Moreover, we assume that for each node, the speed $v$ is uniformly distributed in $\left[0, v_{\max }\right]$, and the angular direction $\theta$ is uniformly distributed in $[0,2 \pi)$. The DP mobility model corresponds, in this case, to the random waypoint mobility model [37].

In Fig. 7, the BER performance in the case of ONRBS is shown as a function of the node spatial density $\rho_{\mathrm{S}}$. In particular, we consider a network-communication scenario with $N=10^{3}$ nodes $\left(\bar{n}_{\mathrm{h}}=18\right)$, and we assume that the message length is $M=10^{4} \mathrm{~b} / \mathrm{msg}$, and that the maximum node speed is $v_{\max }=20 \mathrm{~m} / \mathrm{s}$. As one can observe from the results in Fig. 7, the route BER reaches the floor predicted by the analysis in Section III-A. Note that for $\lambda \leq 0.1 \mathrm{msg} / \mathrm{s}$, the route BER basically coincides with that in the ideal case in the region above the floor. If the traffic load becomes high $(\lambda \geq 1 \mathrm{msg} / \mathrm{s})$, then the route BER is worse than that in the ideal case also above the floor - the route BER is unacceptable anyways. Although the mobility level of the nodes is fairly high, comparing the results in Fig. 7 with the results (not shown here for lack of space) relative to a static ad hoc wireless network, it is immediate to conclude that the difference is negligible. In other words, ONRBS is very robust against node mobility (for sufficiently low interference) and the performance is dictated by the interference level. 


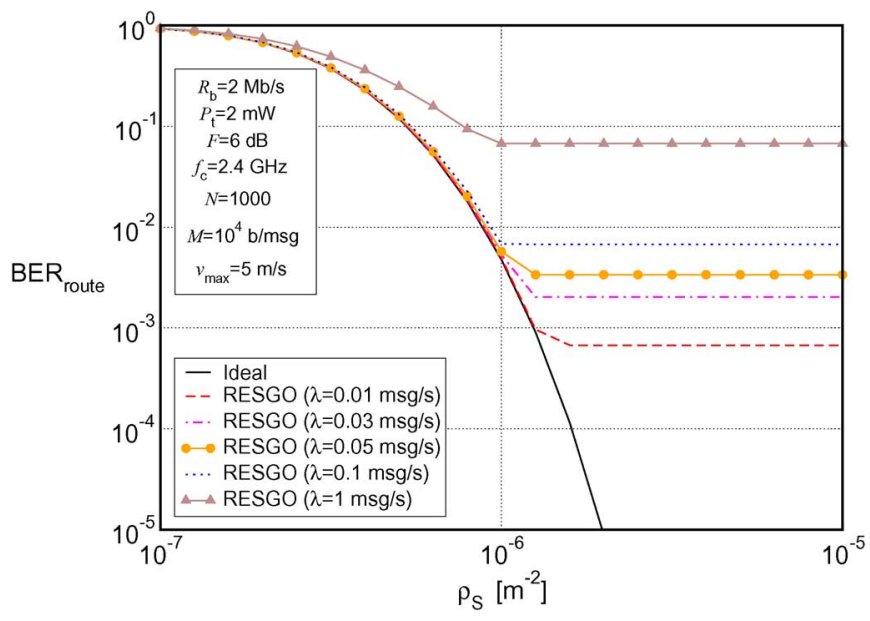

Fig. 8. Route BER versus node spatial density in the case of DP mobility model and RBS. The maximum node speed is $v_{\max }=5 \mathrm{~m} / \mathrm{s}$. The ideal (no INI) case and the realistic (INI) case with RESGO MAC protocol (for various values of the traffic load) are considered.

Considering an RBS-based wireless network-communication scenario, the performance results, in terms of route BER versus node spatial density, are shown in Fig. 8, for $v_{\max }=5 \mathrm{~m} / \mathrm{s}$. Note that the trend of the performance results is very similar to that observed in the ONRBS case shown in Fig. 7. Although the mobility level in the case with $\operatorname{RBS}\left(v_{\max }=5 \mathrm{~m} / \mathrm{s}\right)$ is significantly lower than that in the case with ONRBS $\left(v_{\max }=\right.$ $20 \mathrm{~m} / \mathrm{s}$ ). As in the case with ONRBS, the route BER performance is basically dictated by the interference level. This leads to the following important observation: if the MAC protocol is not effective in canceling or mitigating the interference (as in the case of RESGO), then the performance of an ad hoc wireless network with mobile nodes is likely to be determined by the multiple access interference, rather than the mobility level of the nodes.

A general comment, regarding the BER performance obtained with RBS, might be worthwhile here. In fact, the obtained results are based on the transmission of a single message along the multihop route. However, in packet-switched networks a message could be split into several packets (called datagrams). If this is the case, then the BER performance with RBS could significantly degrade. On the other hand, the performance with ONRBS is basically independent of the number of packets transmitted from source to destination, since each consecutive packet will be opportunistically forwarded by selecting the most convenient route. This underlines the importance, from a practical viewpoint, of a very efficient route maintenance mechanism in an ONRBS-based ad hoc wireless network-communication scenario.

In order to further understand the impact of the speed on the BER performance, in Fig. 9, the BER is evaluated as a function of the maximum speed $v_{\max }$ in ideal and realistic scenarios with RESGO MAC protocol. Two possible values for the average packet transmission rate $(\lambda=0.0001 \mathrm{msg} / \mathrm{s}$ and $\lambda=0.001 \mathrm{msg} / \mathrm{s}$ ) are considered, and the message length is set to $M=10^{6} \mathrm{~b} / \mathrm{msg}(125 \mathrm{kB} / \mathrm{msg})$. The node spatial density is fixed to $\rho_{\mathrm{S}}=0.02 \mathrm{~m}^{-2}$, and the considered number of nodes is $N=10^{3}$. As one can see, the impact of the routing strategy

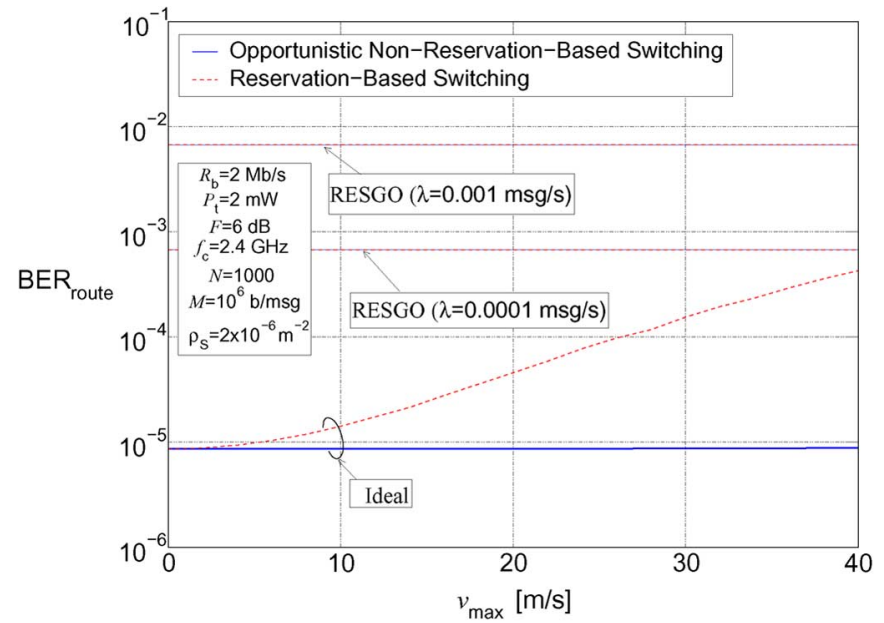

Fig. 9. Route BER versus maximum node speed in the case of DP mobility model. The ideal (no INI) case and the realistic (INI) case with RESGO MAC protocol (for various values of the traffic load) are considered.

(either ONRBS or RBS) is significant only in the ideal case (where there is no interference), whereas there is no performance difference in the realistic (with interference) scenarios. In the ideal case, for increasing values of the maximum speed $v_{\text {max }}$, while the BER in the ONRBS case does not change (the maximum speed should be increased much more to observe a noticeable performance degradation), the BER in the RBS case degrades rapidly, increasing almost proportionally to $v_{\max }$. In fact, an RBS scheme is characterized by the fact that nodes do not change direction of movement during the entire duration of the message transmission, which is equal (neglecting the propagation time) to the product between the message duration $\left(D_{\mathrm{msg}}\right)$ and the number of hops $\left(\bar{n}_{\mathrm{h}}=\bar{\Theta}(\sqrt{N})\right)$. Therefore, if the maximum speed is large, it follows that the nodes of the last links of a route can move very far from each other, with respect to their original positions, so that the BER at the end of a route may significantly degrade. On the other hand, in the case of ONRBS, each link is opportunistically activated, and the corresponding nodes do not move significantly, for all considered values of the maximum speed, during a message transmission of duration $D_{\mathrm{msg}}$. In a realistic network-communication scenario with interference, the impact of mobility is irrelevant (for the considered network conditions). As previously observed, in general, one can conclude that the higher the interference between the nodes (INI), the lower is the impact of the routing strategy on the performance.

In Fig. 10, the dependence of the route BER on the message length is shown in the case of very low node mobility level. More precisely, the maximum speed is $v_{\max }=2 \mathrm{~m} / \mathrm{s}$, which corresponds to a pedestrian network-communication scenario. As one can observe, for increasing message length (i.e., transmission duration), the route BER reaches 1, i.e., the performance becomes unacceptable. While in the ideal case, there is a significant difference between the performance with ONRBS and RBS (for example, at a maximum tolerable route BER equal to $10^{-3}$, the maximum message length supported with ONRBS is $M \simeq 6 \times 10^{8} \mathrm{~b} / \mathrm{msg}$, whereas that supported with RBS is $M \simeq 2.5 \times 10^{7} \mathrm{~b} / \mathrm{msg}$ ), in a realistic case (with 


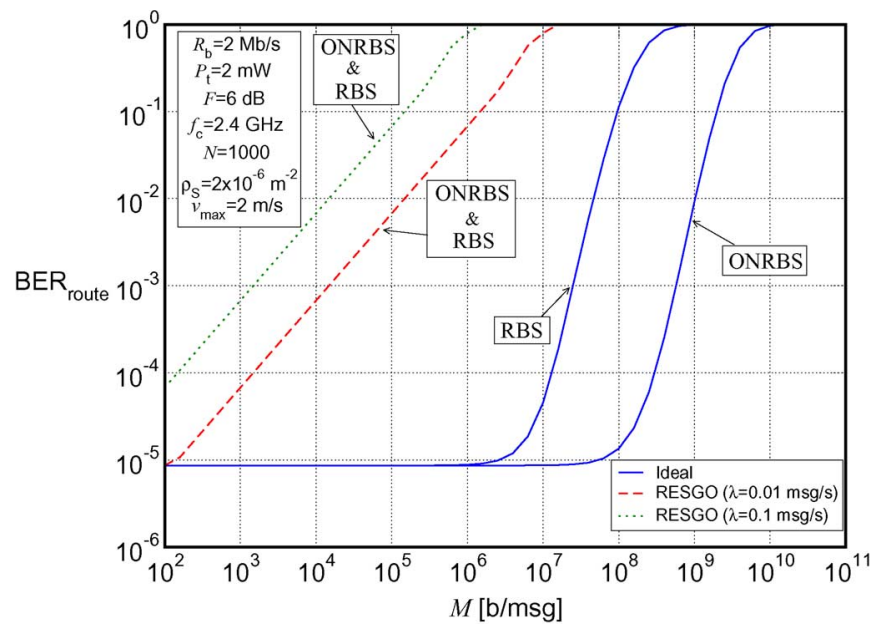

Fig. 10. BER performance versus message dimension $M$ in the case of DP mobility model. The maximum speed is $v_{\max }=2 \mathrm{~m} / \mathrm{s}$. The ideal (no INI) case and realistic case (with INI and various traffic loads) are considered.

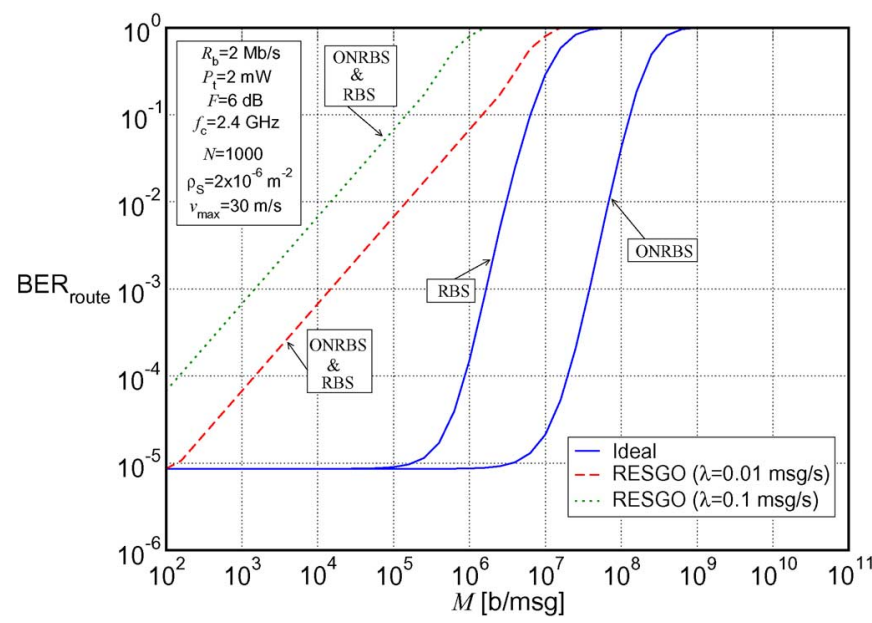

Fig. 11. Route BER performance versus message dimension $M$ in the case of DP mobility model. The maximum speed is $v_{\max }=30 \mathrm{~m} / \mathrm{s}$. The ideal (no INI) case and realistic (INI) case with RESGO MAC protocol (for various traffic loads) are considered.

interference and average packet generation rate $\lambda$ equal to 0.01 and $0.1 \mathrm{msg} / \mathrm{s}$, respectively), there is no difference between ONRBS and RBS-this is also predicted by the results, for low value of $v_{\max }$, shown in Fig. 9. In Fig. 11, the same analysis is conducted in the case with $v_{\max }=30 \mathrm{~m} / \mathrm{s}$ (a vehicular networkcommunication scenario). It is possible to show that an increase of the maximum speed has a dual effect.

1) For a given maximum acceptable BER, in an ideal scenario (without interference), the maximum acceptable message length becomes significantly lower.

2) There is still no performance difference between the two considered switching techniques in a realistic networkcommunication scenario (with interference). These results are in agreement with those in Fig. 9, where the impact of the switching technique is negligible up to $v_{\max }=40 \mathrm{~m} / \mathrm{s}$.

The results in Fig. 11 show that in a realistic scenario with high interference, the two switching schemes offer basically

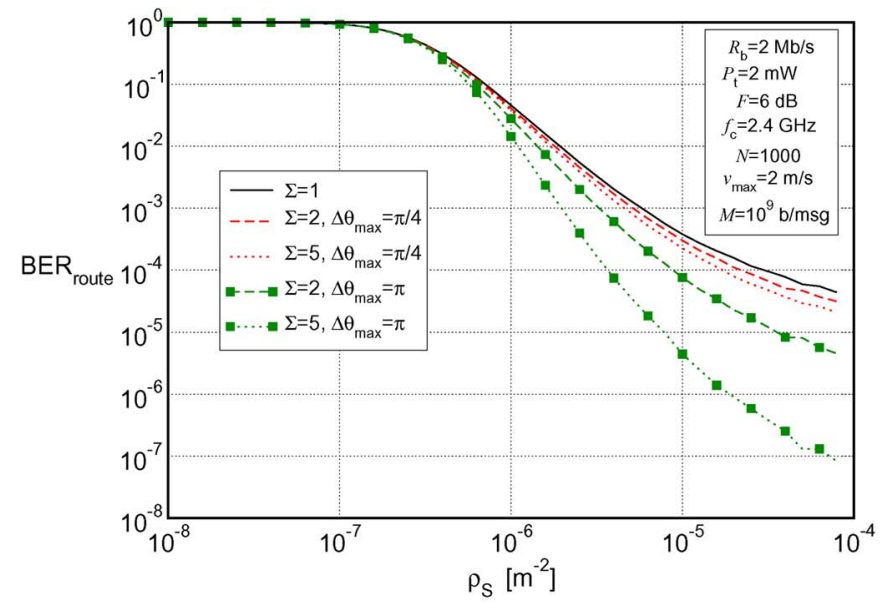

(a)

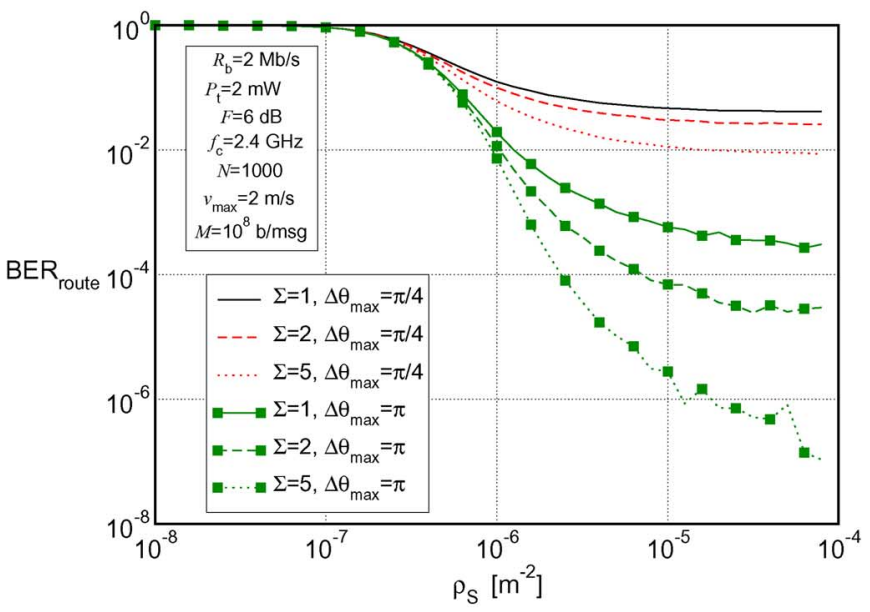

(b)

Fig. 12. Route BER performance versus node spatial density $\rho_{S}$ for a DNP mobility model with (a) ONRBS (the message length is $M=10^{9} \mathrm{~b} / \mathrm{msg}$ ) and (b) RBS (the message length is $M=10^{8} \mathrm{~b} / \mathrm{msg}$ ). Various values of the parameter $\sigma$ and the maximum angle deviation $\Delta \theta_{\max }$ are considered.

the same performance. This underlies the importance of the MAC protocol employed in an ad hoc wireless network. If the MAC protocol is not effective in combating the interference, then switching strategy entails minor performance differences. In other words, the switching strategy (or routing) plays an important role only if the MAC protocol is effective in mitigating the interference in the network.

\section{B. DNP Mobility Model}

As mentioned in Section V-B, it is intuitively obvious that the faster a node is moving, the less pronounced is the direction change during a message transmission. In order to formalize this intuition, we assume that the direction change $\Delta \theta$ between two consecutive time slots can be written as

$$
\Delta \theta= \pm \Delta \theta_{\max }\left(1-e^{-\frac{1}{v}}\right)
$$

where $v$ is the speed of the node (constant for the entire message transmission), $\Delta \theta_{\max }$ corresponds to the maximum considered 


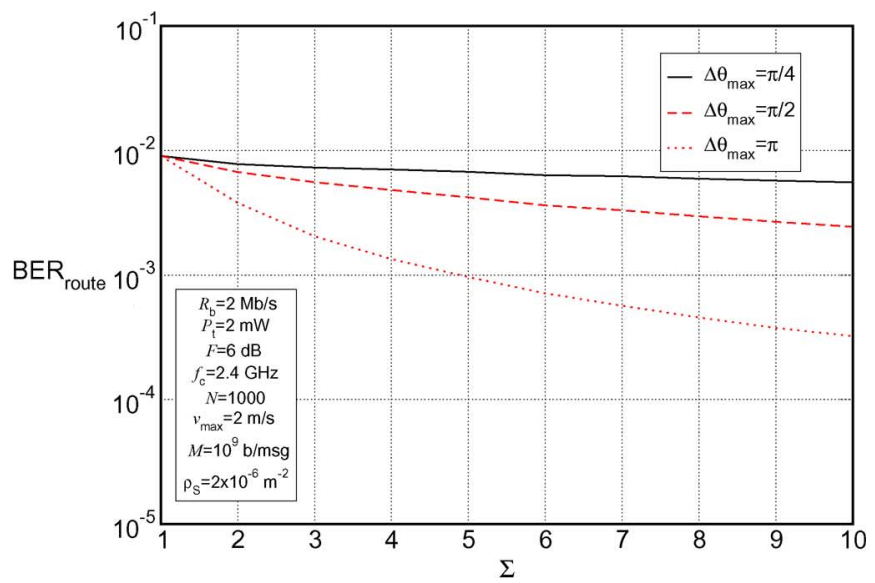

(a)

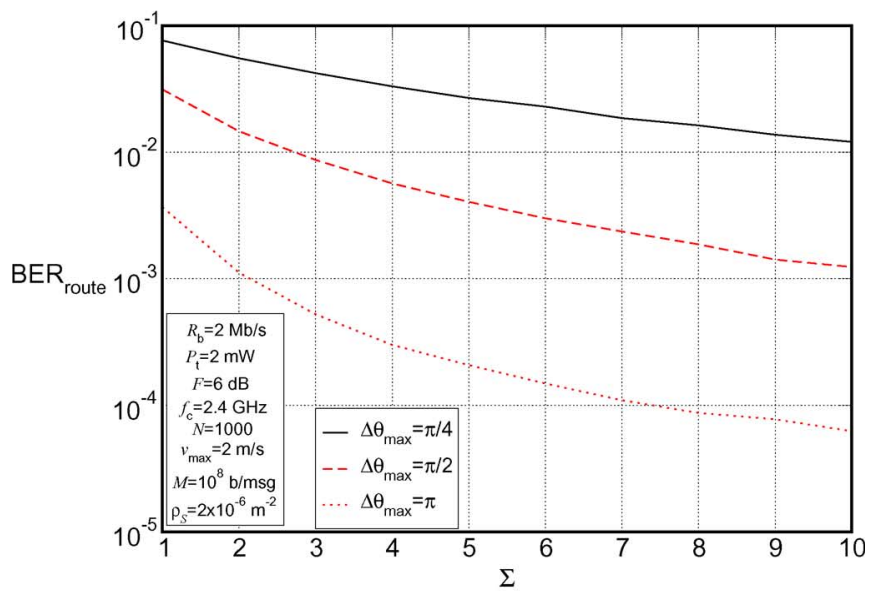

(b)

Fig. 13. Route BER performance versus parameter $\Sigma$ in the case of DNP mobility model with (a) ONRBS (the message length is $M=10^{9} \mathrm{~b} / \mathrm{msg}$ ) and (b) RBS (the message length is $M=10^{8} \mathrm{~b} / \mathrm{msg}$ ). Various values of the maximum angle deviation $\Delta \theta_{\max }$ are considered.

change of angular direction of movement, and the sign in front of the angular deviation ( + or - ) is chosen randomly and independently in successive slots. In order to evaluate the impact of the proposed DNP mobility model with respect to that of the DP mobility model, the following analysis is limited to an ideal (no INI) case. The extension to a realistic (INI) case is straightforward. However, the maximum speed is set to $v_{\max }=2 \mathrm{~m} / \mathrm{s}$ in all considered examples: this corresponds to a pedestrian network-communication scenario and justifies multiple changes of direction during a message transmission.

In Fig. 12, the route BER performance is shown as a function of the node spatial density, considering ONRBS [Fig. 12(a)] and RBS [Fig. 12(b)]. All the major network parameters, except for the message length, have the same values in both cases. In fact, the message length is $M=10^{9} \mathrm{~b} / \mathrm{msg}$ with ONRBS, whereas it is $M=10^{8} \mathrm{~b} / \mathrm{msg}$ with RBS. In both cases, three possible values for the parameter $\Sigma(1,2$, and 5$)$ and two possible values for the maximum angular deviation $\Delta \theta_{\max }(\pi / 4$ and $\pi)$ are considered. It is immediate to notice that an increase of $\Sigma$ and/or $\Delta \theta_{\max }$ has a beneficial effect on the route BER performance. In particular, the performance improvement is much more pronounced in the case with RBS. In fact, in this

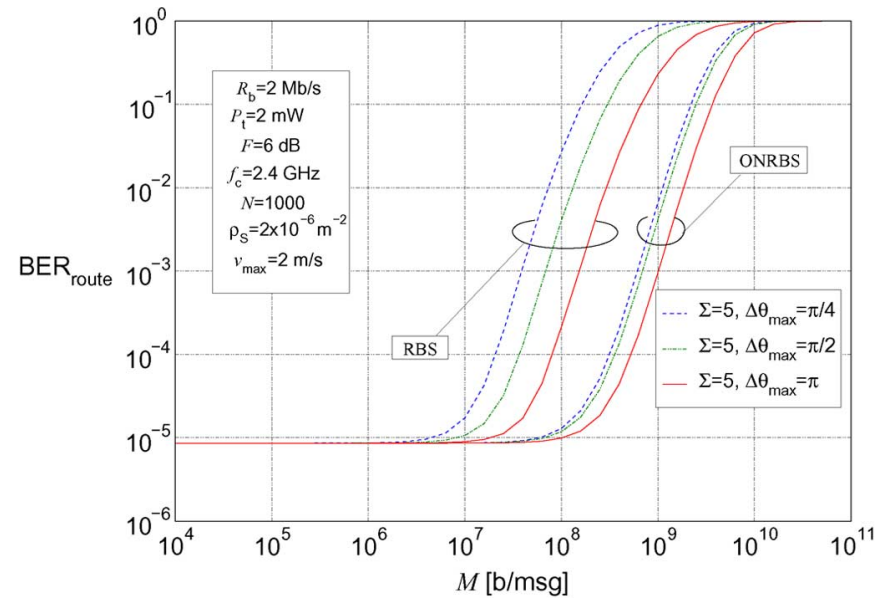

Fig. 14. BER performance versus parameter $M$ in the case of DNP mobility model. The performance for both the cases with ONRBS and RBS is shown. The node spatial density is $\rho_{S}=2 \times 10^{-6} \mathrm{~m}^{-2}$ and the maximum speed is $v_{\max }=2 \mathrm{~m} / \mathrm{s}$. Various values of the parameter $\Sigma$ and the maximum angle deviation $\Delta \theta_{\max }$ are considered.

case, the latest links of the route, rather than tending to become longer, do not change significantly, since the frequent direction changes force a node to move around its original position. One can also observe that in the case with $\Sigma=1$, while the performance with ONRBS is independent of $\Delta \theta_{\max }$ (since each link has initial length equal to $\left.1 / \sqrt{\rho_{S}}\right)$, the performance with RBS is not.

The impact of the number $\Sigma$ of direction changes on the BER performance is shown in Fig. 13 in the cases with ONRBS $\left(M=10^{9} \mathrm{~b} / \mathrm{msg}\right)$ [Fig. 13(a)] and RBS $\left(M=10^{8} \mathrm{~b} / \mathrm{msg}\right)$ [Fig. 13(b)]. As one can see, increasing values of $\Sigma$ improve the performance. However, the relative performance improvement is more evident for low values of $\Sigma$, while it reduces for larger values. As noticed for the results in Fig. 12, in this case as well, it is evident that the performance improvement, for increasing values of the parameter $\Sigma$, is more pronounced in RBS-based schemes than in ONRBS-based schemes.

Finally, in Fig. 14, the BER performance, as a function of the message length $M$, is shown in both ONRBS- and RBS-based schemes. The parameter $\Sigma$ is fixed to 5 , and various values of the maximum angular deviation $\Delta \theta_{\max }$ are considered. The qualitative behavior of the performance curves is the same as that shown in Fig. 10, computed with the DP mobility model. As previously noticed, increasing the maximum angular deviation $\Delta \theta_{\max }$ has a beneficial effect, especially in an RBS-based ad hoc wireless network. In other words, stronger changes of direction alleviate the degradation brought about by node mobility, and this effect is more pronounced in an ad hoc wireless network-communication scenario where there is no adaptive route maintenance.

\section{CONCLUSION}

Many factors affect the performance of ad hoc wireless networks, among which node mobility plays a significant role. While routing is usually studied by assuming perfect connectivity, in this paper we have proposed a semi-analytical approach to investigate the relation between node mobility, 
switching strategy, and physical-layer characteristics, and their impact on the BER performance at the end of a multihop route. The proposed framework can be used in conjunction with any mobility model, provided that a suitable statistical description is available. Our results show that the use of ONRBS allows supporting, at the expense of heavier control traffic, a higher mobility level than the use of RBS. We have also shown that the larger the traffic load (and, consequently, the interference), the lower is the impact of the routing (or switching) strategy (i.e., RBS versus ONRBS) on the network performance. Two mobility models, namely DP and DNP, have been considered. Our results show that, in RBS-based ad hoc wireless networks, DNP mobility supports a better performance than DP mobility, since frequent changes of directions average out, forcing the nodes to move around their original positions, rather than moving far away and, therefore, disrupting connectivity.

Finally, a general conclusion of this work is that switching (and, therefore, routing) plays an important role in ad hoc wireless networks only if the MAC protocol is effective against the interference. If communications in the network are affected by significant interference, then the choice of the switching scheme does not and cannot significantly improve the performance.

\section{ACKNOWLEDGMENT}

The authors would like to thank the anonymous reviewers of this paper, whose insightful comments helped improve the quality of this paper substantially.

\section{REFERENCES}

[1] C. E. Perkins, Ad Hoc Networking. Upper Saddle River, NJ: AddisonWesley, 2001.

[2] C.-K. Toh, Ad Hoc Mobile Wireless Networks. Upper Saddle River, NJ: Prentice-Hall, 2002.

[3] C. E. Perkins and P. Bhagwat, "Highly dynamic destination-sequenced distance-vector routing (DSDV) for mobile computers," Comput. Commun. Rev., vol. 24, no. 4, pp. 234-244, Oct. 1994.

[4] D. B. Johnson and D. A. Maltz, "Dynamic source routing in ad hoc wireless networks," in Mobile Computing, T. Imielinski and H. Korth, Eds. Norwell, MA: Kluwer, 1996, pp. 153-181.

[5] C. E. Perkins and E. M. Royer, "Ad hoc on-demand distance vector routing," in Proc. IEEE Workshop Mobile Comput. Syst. and Appl., New Orleans, LA, Feb. 1999, pp. 90-100.

[6] S. Park and B. Van Voorst, "Anticipated route maintenance (ARM) in location-aided mobile ad hoc networks," in Proc. IEEE GLOBECOM, San Antonio, TX, Nov. 2001, pp. 2809-2813.

[7] W. Su, S.-J. Lee, and M. Gerla, "Mobility prediction in wireless networks," in Proc. IEEE MILCOM, Los Angeles, CA, Oct. 2000, pp. 491-495.

[8] G. Lim, K. Shin, S. Lee, H. Yoon, and J. S. Ma, "Link stability and route lifetime in ad hoc wireless networks," in Proc. ICPPW, Vancouver, BC, Canada, Aug. 2002, pp. 116-123.

[9] D. Son, A. Helmy, and B. Krishnamachari, "The effect of mobilityinduced location errors on geographic routing in mobile ad hoc and sensor networks: Analysis and improvement using mobility prediction," IEEE Trans. Mobile Comput., vol. 3, no. 3, pp. 233-245, Jul.-Sep. 2004.

[10] R. Rao and G. Kesidis, "Purposeful mobility for relaying and surveillance in mobile ad hoc sensor networks," IEEE Trans. Mobile Comput., vol. 3, no. 3, pp. 225-232, Jul.-Sep. 2004.

[11] P. Gupta and P. R. Kumar, "The capacity of wireless networks," IEEE Trans. Inf. Theory, vol. 46, no. 2, pp. 388-404, Mar. 2000.

[12] M. Grossglauser and D. Tse, "Mobility increases the capacity of ad hoc wireless networks," IEEE/ACM Trans. Netw., vol. 10, no. 4, pp. 477-486, Aug. 2002.
[13] D. D. Perkins, H. D. Hughes, and C. B. Owen, "Factors affecting the performance of ad hoc networks," in Proc. IEEE ICC, New York, Apr. 2002, pp. 2048-2052.

[14] M. J. Neely and E. Modiano, "Capacity and delay tradeoffs for ad hoc mobile networks," IEEE Trans. Inf. Theory, vol. 51, no. 6, pp. 1917-1937, Jun. 2005.

[15] S. Toumpis and A. J. Goldsmith, "Large wireless networks under fading, mobility and delay constraints," in Proc. IEEE Conf. Comput. Commun. (INFOCOM), 2004, pp. 609-619.

[16] G. Ferrari and O. K. Tonguz, "MAC protocols and transport capacity in ad hoc wireless networks: Aloha versus PR-CSMA," in Proc. IEEE Military Commun. Conf. (MILCOM), Boston, MA, Oct. 2003, vol. 2, pp. $1311-1318$.

[17] 1 , "Performance of circuit-switched ad hoc wireless networks with Aloha and PR-CSMA MAC protocols," in Proc. IEEE Global Telecommun. Conf. (GLOBECOM), San Francisco, CA, Dec. 2003, pp. 2824-2829.

[18] N. Wisitpongphan and O. K. Tonguz, "Disjoint multi-path source routing in ad hoc networks: Transport capacity," in Proc. IEEE VTC-Fall, Orlando, FL, Oct. 2003, pp. 2207-2211.

[19] O. K. Tonguz and G. Ferrari, Ad Hoc Wireless Networks: A Communication-Theoretic Perspective. Hoboken, NJ: Wiley, 2006.

[20] N. Abramson, "The throughput of packet broadcasting channels," IEEE Trans. Commun., vol. COM-25, no. 1, pp. 117-128, Jan. 1977.

[21] S. Panichpapiboon, G., Ferrari, N. Wisitpongphan, O. K. Tonguz, "Route reservation in ad hoc networks: Is it a good idea?" in Proc. IEEE Wireless Communication and Networking Conference, New Orleans, LA, Mar. 2005, pp. 2045-2050.

[22] _ "Route reservation in ad hoc wireless networks: Is it a good idea?" IEEE Trans. Mobile Comput., vol. 6, no. 1, Jan. 2007.

[23] T. H. Cormen, C. E. Leiserson, R. L. Rivest, and C. Stein, Introduction to Algorithms, 2nd ed. Cambridge, MA: MIT Press, 2002.

[24] T. S. Rappaport, Wireless Communications. Principles \& Practice, 2nd ed. Upper Saddle River, NJ: Prentice-Hall, 2002.

[25] G. Ferrari, B. Baruffini, and O. K. Tonguz, "Spectral efficiencyconnectivity tradeoff in ad hoc wireless networks," in Proc. IEEE Symp. Inf. Theory and Appl. (ISITA), Parma, Italy, Oct. 2004, pp. 451-456.

[26] A. Papoulis, Probability, Random Variables and Stochastic Processes. New York: McGraw-Hill, 1991.

[27] L. Kleinrock and F. A. Tobagi, "Packet switching in radio channels: Part I-Carrier sense multiple-access modes and their throughput-delay characteristics," IEEE Trans. Commun., vol. COM-23, no. 12 , pp. $1400-1416$, Dec. 1975.

[28] F. A. Tobagi and L. Kleinrock, "Packet switching in radio channels: Part II-The hidden terminal problem in carrier sense multiple-access and the busy-tone solution," IEEE Trans. Commun., vol. COM-23, no. 12, pp. 1417-1433, Dec. 1975

[29] G. Ferrari, O. K. Tonguz, and M. Bhatt, "Impact of receiver sensitivity on the performance of sensor networks," in Proc. IEEE VTC_Spring, Milan, Italy, Apr. 2004, pp. 1350-1354.

[30] S. Panichpapiboon, G. Ferrari, and O. K. Tonguz, "Sensor networks with random versus uniform topology: MAC and interference considerations," in Proc. IEEE VTC, Milan, Italy, Apr. 2004, pp. 2111-2115.

[31] N. Wisitpongphan, G. Ferrari, S. Panichpapiboon, J. S. Parikh, and O. K. Tonguz, "QoS provisioning using BER-based routing for ad hoc wireless networks," in Proc. IEEE VTC, Stockholm, Sweden, May 2005, pp. 2483-2487.

[32] Y.-C. Cheng and T. G. Robertazzi, "Critical connectivity phenomena in multihop radio models," IEEE Trans. Commun., vol. 37, no. 7, pp. 770-777, Jul. 1989.

[33] C. Schurgers, V. Tsiatsis, S. Ganeriwal, and M. Srivastava, "Topology management for sensor networks: Exploiting latency and density," in Proc. ACM Int. Symp. MOBIHOC, Lausanne, Switzerland, Jun. 2002, pp. $135-145$.

[34] Institute of Electrical and Electronics Engineers, IEEE Std 802.11b1999/Cor 1-2001. Part 11: Wireless LAN Medium Access Control (MAC) and Physical Layer (PHY) Specifications, 2001.

[35] L. M. Feeney, M. Nilsson, "Investigating the energy consumption of a wireless network interface in an ad hoc networking environment," in Proc. IEEE Conf. Comput. Commun. Anchorage, AK: INFOCOM, Apr. 2001, pp. $1548-1557$

[36] Smart Dust Project at UC Berkeley, Autonomous Sensing and Communication in a Cubic Millimeter. [Online]. Available: http://robotics.eecs. berkeley.edu/ pister/SmartDust

[37] T. Camp, J. Boleng, and V. Davies, "A survey of mobility models for ad hoc network research," Wirel. Commun. Mobile Comput. (WCMC)—Special Issue Mobile Ad Hoc Networking: Research, Trends and Applications, vol. 2, no. 5, pp. 483-502, 2002. 


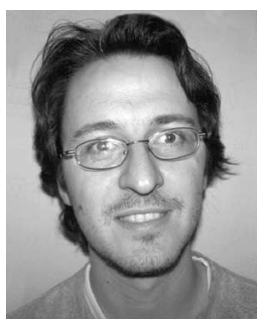

Gianluigi Ferrari (S'97-M'03) was born in Parma, Italy, in November 1974. He received the "Laurea" degree (five-year program) (summa cum laude) and the $\mathrm{Ph} . \mathrm{D}$. degree in electrical engineering from the University of Parma in October 1998 and January 2002, respectively.

From July 2000 to December 2001, he was a Visiting Scholar at the Communication Sciences Institute, University of Southern California, Los Angeles. Since 2002, he has been a Research Professor with the Department of Information Engineering, University of Parma, where he is now the coordinator of the Wireless Ad-hoc and Sensor Networks (WASN) Laboratory. Between 2002 and 2004, he visited several times, as a Research Associate, the Electrical and Computer Engineering Department at Carnegie Mellon University, Pittsburgh, PA. He has published more than 70 papers in leading international conferences and journals. He is coauthor of the books Detection Algorithms for Wireless Communications, with Applications to Wired and Storage Systems (Wiley: 2004), Teoria della probabilità e variabili aleatorie con applicazioni (McGraw-Hill: 2005), and Ad Hoc Wireless Networks: A Communication-Theoretic Perspective (Wiley: 2006). He is listed in Marquis Who's Who in the World, Who's Who in Science and Engineering, Who's Who of Emerging Leaders, Madison Who's Who, and 2000 Outstanding Intellectuals of the 21st Century, International Biographical Centre, U.K., 2005. His research interests include digital communication systems design, adaptive signal processing (with particular emphasis on iterative detection techniques for channels with memory), information theory, and ad hoc wireless networking.

Dr. Ferrari is a co-recipient of a best student paper award at the 2006 International Workshop on Wireless Ad hoc Networks (IWWAN'06). He acts as a frequent reviewer for many international journals and conferences. He acts also as a technical program member for several international conferences (IEEE ICC, IEEE WirelessCom, IEEE IWCMC, IEEE WCNC, Accessnets, IWWAN, IASTED CSA, IASTED WOC, IEEE SECON, IASTED CSNA, and SympoTIC).

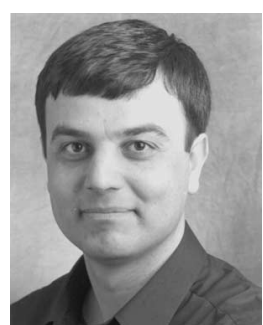

Ozan K. Tonguz (S'86-M'90) was born in Nicosia, Cyprus. He received the B.Sc. degree from the University of Essex, Colchester, U.K., in 1980 and the M.Sc. and Ph.D. degrees from Rutgers University, New Brunswick, NJ, in 1986, and 1990, respectively, all in electrical engineering.

He currently serves as a Tenured Full Professor with the Department of Electrical and Computer Engineering, Carnegie Mellon University (CMU), Pittsburgh, PA. Before joining CMU in August 2000, he was with the Electrical and Computer Engineering Department, State University of New York at Buffalo (SUNY/Buffalo). He joined SUNY/Buffalo in 1990 as an Assistant Professor, where he was granted early tenure. He was promoted to Associate Professor in 1995 and to Full Professor in 1998. Prior to joining academia, he was with Bell Communications Research (Bellcore) between 1988 and 1990, doing research on optical networks and communication systems. His current research interests are in wireless networks and communication systems, high-speed networking (Internet), optical networks, satellite communications, bioinformatics, and security. He has published over 200 technical papers in IEEE journals and conference proceedings. He is well known for his contributions to optical networks (optical transmission-access-networking; especially radio over fiber networks, coherent lightwave transmission systems, and amplified direct-detection lightwave transmission systems) and wireless communications and networks (physical layer communications, access, and networking). His recent work on the Integrated Cellular and Ad Hoc Relay Systems (iCAR) is internationally acclaimed as well (the work has received more than 200 citations in about four years). He is the author of the bestseller Ad Hoc Wireless Networks: A Communication-Theoretic Perspective (Wiley: 2006). He was also the architect of the "High Performance Waveform (HPW)" that was implemented in Harris RF Communications' AN/PRC-117f UHF band man-pack tactical radio. His industrial experience includes periods with Bell Communications Research, CTI, Inc., Harris RF Communications, Aria Wireless Systems, Clearwire Technologies, Nokia Networks, Nokia Research Center, Neuro Kinetics, Asea Brown Boveri (ABB), General Motors (GM), and Intel. He currently serves as or has served as a consultant for several companies (such as Aria Wireless Systems, Harris RF Communications, Clearwire Technologies, Nokia Networks, Alcatel and Lucent Technologies), law firms, and government agencies in the United States and Europe in the broad area of telecommunications and networking. He is also a Co-Director (Thrust Leader) of the Center for Wireless and Broadband Networking Research at CMU. More details about his research interests, research group, projects, and publications can be found at http://www.ece.cmu.edu/ tonguz/.

Dr. Tonguz currently serves or has served as an Associate Editor for the IEEE TRANSACTIONS ON COMMUNICATIONS, IEEE COMMUNICATIONS MAGAZINE, and IEEE JOURNAL OF LightwAVE TECHNOLOGY, in addition to serving on the Technical Program Committees of several IEEE conferences [such as INFOCOM, GLOBECOM, ICC, Vehicular Technology Conference (VTC), WCNC], and symposia in the area of wireless communications and optical networks. He was a Guest Editor of the special issue of the IEEE JOURNAL OF LIGHTWAVE TECHNOLOGY and IEEE JOURNAL ON SELECTED AREas In COMmUnications on Multiwavelength Optical Networks and Technology, published in 1996, and a Guest Editor of the Special Issue of the Journal of Mobile Multimedia on Advanced Mobile Technologies for Health Care Applications in 2006. 